\title{
Variational Bayesian Super Resolution
}

\author{
S. Derin Babacan, Member, IEEE, Rafael Molina, Member, IEEE, and Aggelos K. Katsaggelos, Fellow, IEEE
}

\begin{abstract}
In this paper, we address the super resolution (SR) problem from a set of degraded low resolution (LR) images to obtain a high resolution (HR) image. Accurate estimation of the sub-pixel motion between the LR images significantly affects the performance of the reconstructed HR image. In this paper, we propose novel super resolution methods where the HR image and the motion parameters are estimated simultaneously. Utilizing a Bayesian formulation, we model the unknown HR image, the acquisition process, the motion parameters and the unknown model parameters in a stochastic sense. Employing a variational Bayesian analysis, we develop two novel algorithms which jointly estimate the distributions of all unknowns. The proposed framework has the following advantages: 1) Through the incorporation of uncertainty of the estimates, the algorithms prevent the propagation of errors between the estimates of the various unknowns; 2) the algorithms are robust to errors in the estimation of the motion parameters; and 3) using a fully Bayesian formulation, the developed algorithms simultaneously estimate all algorithmic parameters along with the HR image and motion parameters, and therefore they are fully-automated and do not require parameter tuning. We also show that the proposed motion estimation method is a stochastic generalization of the classical Lucas-Kanade registration algorithm. Experimental results demonstrate that the proposed approaches are very effective and compare favorably to state-of-the-art SR algorithms.
\end{abstract}

Index Terms-Bayesian methods, parameter estimation, super resolution, total variation, variational methods.

\section{INTRODUCTION}

I $\mathrm{N}$ many imaging applications, acquiring an image of a scene with high spatial resolution is not possible due to a number of theoretical and practical limitations. These limitations include for instance the sensor resolution, the Rayleigh resolution limit, the increased cost, data transfer rate and the amount of shot noise due to the size of the digital sensor, among others. In these cases, super resolution (SR) methods can be utilized to process one or more low-resolution (LR) images of the scene together to obtain a high-resolution (HR) image. The basic principle of super resolution is that changes in the LR images caused

Manuscript received October 27, 2009; revised April 30, 2010, August 12, 2010; accepted September 06, 2010. Date of publication September 27, 2010; date of current version March 18, 2011. This work was supported in part by a grant from the Department of Energy (DE-NA0000457) and the "Comisión Nacional de Ciencia y Tecnología" under contract TIC2007-65533 and the Spanish research programme Consolider Ingenio 2010: MIPRCV (CSD2007-00018). The associate editor coordinating the review of this manuscript and approving it for publication was Dr. Arun Ross.

S. Derin Babacan is with the Beckman Institute for Advanced Science and Technology, University of Illinois at Urbana-Champaign, IL 61801 USA (e-mail: dbabacan@illinois.edu).

R. Molina is with the Departamento de Ciencias de la Computación e I.A. Universidad de Granada, Spain (e-mail: rms@ decsai.ugr.es).

A. K. Katsaggelos is with the Department of Electrical Engineering and Computer Science, Northwestern University, IL 60208-3118 USA (e-mail: aggk@eecs.northwestern.edu).

Color versions of one or more of the figures in this paper are available online at http://ieeexplore.iee.org.

Digital Object Identifier 10.1109/TIP.2010.2080278 by the blur and the (camera or scene) motion provide additional data that can be utilized to reconstruct the HR image from the set of LR observations. Super resolution methods are widely utilized in a number of imaging fields, such as surveillance, remote sensing, medical and nano-imaging.

Although the super resolution literature is rich (see [1]-[3] for reviews) it is still an open and widely investigated topic. Super resolution methods utilizing a set of LR images generally consist of two parts: Registration, where the motion between the LR images is estimated; and image estimation, where the HR image is recovered from the LR images using information about the motion and blurring. Many conventional methods in the literature assume that the motion information is known $a$ priori. However, this assumption does not hold in many practical systems since exact motion information is very hard to obtain. Therefore, a registration step is needed to obtain the motion parameters from the LR images.

Super resolution is a highly ill-posed problem, especially when the motion parameters are estimated along with the HR image solely from the LR images. The registration parameters are generally very hard to estimate using only LR observations, which makes estimation errors unavoidable in many practical systems. The errors in estimating the registration parameters cause significant drawbacks in super resolution, leading to instabilities in the recovery of the HR image and significantly affecting the robustness of the restoration procedures.

A number of approaches have been proposed to address this problem, which can be classified into two major categories based on the stage where the registration is performed. The first class of methods employ registration as a preprocessing stage [4]-[7]. The motion parameters are estimated from the observed LR images, and then used in a separate image estimation process. Since the motion parameters estimated using only the LR images can be unreliable, a desired property of the algorithm is robustness to outliers and errors in motion estimates. A robust backprojection method is proposed in [5] based on median estimators. Farsiu et al. [6] proposed to use an observation model based on $l_{1}$-norms and image priors based on bilateral total-variation (BTV) functions, whose combination makes the algorithm robust to motion outliers. Other methods employ regularization by modeling the registration errors as Gaussian noise [8], [9]. All methods in this category attempt to reduce the effect of estimation errors and noise by decreasing the weight of unreliable observations in the restoration process, but they do not attempt to correct the errors in the motion estimation process.

Another class of SR methods estimate both the HR image and the motion parameters simultaneously. The most common approach in this category is alternating minimization (AM), where at each iteration, the estimates of the HR image and the motion parameters are improved progressively in an alternating fashion [10]-[20]. Some methods in this category also employ explicit 
models of the errors in motion estimates. In [16] and [17], the errors in motion and blur parameters are assumed to follow Gaussian distributions. In [16], the HR image is marginalized out from the joint distribution and the motion and blur parameters are estimated from this marginal distribution. A major disadvantage of this method is that the marginalization of the HR image requires the utilization of a Gaussian image prior, which overpenalizes strong image edges and therefore reduces the quality of the estimated HR image. In [17], this problem is overcome by marginalizing the motion and blur parameters, and employing a Huber prior to model the HR image. Recently, joint identification methods are proposed in [21]-[23] where the optimization problem is solved simultaneously for both the HR image and motion parameters. Methods which do not utilize explicit knowledge of the motion estimation parameters have been proposed in [24], [25]. Finally, a number of methods have been proposed recently that utilize a single image and example-based learning methods [26], [27].

A major drawback of most super resolution methods is that they employ a number of unknown parameters that need to be tuned. This tuning process can be cumbersome and time-consuming since the parameter values have to be chosen differently for each image and degradation condition. Moreover, the algorithmic performance depends significantly on the appropriate choice of parameters, such that generally a long supervised process is needed to obtain useful results.

In this paper, we propose two novel Bayesian super resolution methods which address both of the above mentioned issues. We provide a systematic modeling of the unknown HR image and the motion parameters within a novel hierarchical Bayesian formulation, and develop SR algorithms which jointly estimate the HR image and the motion. Through the utilization of variational Bayesian analysis, the proposed framework provides uncertainties of the estimates during the restoration process, which helps to prevent error-propagation and improves robustness. All required algorithmic parameters are estimated along with the HR image and the motion parameters, and therefore algorithms do not require user supervision. Moreover, the parameters are estimated optimally in a stochastic sense, which provides high reconstruction performance. We show that the proposed methods are very robust to errors in initial motion estimates due to adaptive parameter and motion estimation. We demonstrate with experimental results that the proposed methods provide HR images with high quality and accurate motion information, and compare favorably to existing SR methods.

The rest of this paper is organized as follows. Section II provides the mathematical model for the LR image acquisition process. We provide the description of the hierarchical Bayesian framework modeling the unknowns in Section III. The inference procedure to develop the proposed methods is presented in Section IV. We demonstrate the effectiveness of the proposed methods with experimental results in Section V and conclusions are drawn in Section VI.

\section{PROBlem Formulation}

The imaging process is assumed to have generated $L \mathrm{LR}$ images $\mathbf{y}_{k}, k=1, \ldots, L$, from the HR image $\mathbf{x}$. The LR images $\mathbf{y}_{k}$ and the HR image $\mathbf{x}$ consist of $N$ and $P N$ pixels, respectively, where the integer $P>1$ is the factor of increase in resolution (non-integer factors can also be supported by appropriately changing the downsampling and interpolation schemes). In this paper we adopt the matrix-vector notation such that the images $\mathbf{y}_{k}$ and $\mathbf{x}$ are arranged as $N \times 1$ and $P N \times 1$ vectors, respectively. The imaging process introduces shifting, blurring and downsampling, which is modeled as

$$
\mathbf{y}_{k}=\mathbf{A} \mathbf{H}_{k} \mathbf{C}\left(\mathbf{s}_{k}\right) \mathbf{x}+\mathbf{n}_{k}=\mathbf{B}_{k}\left(\mathbf{s}_{k}\right) \mathbf{x}+\mathbf{n}_{k}
$$

where $\mathbf{A}$ is the $N \times P N$ downsampling matrix, $\mathbf{H}_{k}$ is the $P N \times P N$ blurring matrix, $\mathbf{C}\left(\mathbf{s}_{k}\right)$ is the $P N \times P N$ warping matrix generated by the motion vector $\mathbf{s}_{k}$, and $\mathbf{n}_{k}$ is the $N \times 1$ acquisition noise. Note that the matrices $\mathbf{H}_{k}$ and $\mathbf{C}\left(\mathbf{s}_{k}\right)$ and the noise $\mathbf{n}_{k}$ can be different for each LR image $\mathbf{y}_{k}$. In this work, we assume that the blurring matrices $\mathbf{H}_{k}$ are known.

In this work we assume that the motion vectors $\mathbf{s}_{k}$ are not known, so they have to be estimated along with the HR image $\mathrm{x}$. We consider a motion model consisting of translational and rotational motion, so that $\mathbf{s}_{k}=\left(\theta_{k}, c_{k}, d_{k}\right)^{T}$, where $\theta_{k}$ is the rotation angle, and $c_{k}$ and $d_{k}$ are the horizontal and vertical translations of the $k$ th HR image, respectively, with respect to the reference frame $\mathbf{x}$. This motion model is quite general as opposed to many existing SR methods which consider only translational motion. Additionally, as will be shown later, the proposed framework can be extended to more complex motion models such as affine and projective motion (similarly to [22], [23]).

The effects of downsampling, blurring, and warping can be combined into a single $N \times P N$ system matrix $\mathbf{B}_{k}\left(\mathbf{s}_{k}\right)$, such that each row in matrix $\mathbf{B}_{k}\left(\mathbf{s}_{k}\right)$ maps the pixels in the HR image $\mathbf{x}$ to one pixel in the LR image $\mathbf{y}_{k}$. Given (1), the super resolution problem is to find an estimate of the HR image $\mathbf{x}$ from the set of LR images $\left\{\mathbf{y}_{k}\right\}$ using prior knowledge about $\left\{\mathbf{C}\left(\mathbf{s}_{k}\right)\right\}$, $\left\{\mathbf{n}_{k}\right\}$, and $\mathbf{x}$.

\section{HiERARChICAL BAYESIAN Model}

In order to obtain high quality estimates of $\mathbf{x},\left\{\mathbf{s}_{k}\right\}$ and the model parameters from $\left\{\mathbf{y}_{k}\right\}$, properties of the unknowns and the acquisition process have to be taken into account. In Bayesian models, the incorporation of prior knowledge is achieved by treating all unknowns as stochastic quantities and by assigning probability distributions to them. These distributions are used to introduce prior knowledge into the estimation process.

In this work, we adopt a hierarchical Bayesian framework consisting of two stages. The first stage is used to model the acquisition process, the unknown HR image $\mathbf{x}$ and the motion vectors $\left\{\mathbf{s}_{k}\right\}$. The unknowns $\mathbf{x}$ and $\mathbf{s}_{k}$ are assigned prior distributions $\mathrm{p}\left(\mathbf{x} \mid \alpha_{\mathrm{im}}\right)$ and $\mathrm{p}\left(\mathbf{s}_{k}\right)$, respectively. The observation $\mathbf{y}=$ $\left\{\mathbf{y}_{k}\right\}$ is also a random process with the corresponding conditional distribution $\mathrm{p}\left(\mathbf{y} \mid \mathbf{x},\left\{\mathbf{s}_{k}\right\},\left\{\beta_{k}\right\}\right)$. These distributions depend on additional parameters $\alpha_{\mathrm{im}}$ and $\left\{\beta_{k}\right\}$ (called hyperparameters), which are modeled by assigning hyperprior distributions in the second stage of the hierarchical model.

In the following subsections we provide the description of individual distributions used to model the unknowns. 


\section{A. Observation Model}

Using the model in (1) and assuming that $\mathbf{n}_{k}$ is zero-mean white Gaussian noise with the inverse variance (precision) $\beta_{k}$, the conditional distribution of the LR image $\mathbf{y}_{k}$ is given by

$$
\mathrm{p}\left(\mathbf{y}_{k} \mid \mathbf{x}, \mathbf{s}_{k}, \beta_{k}\right) \propto \beta_{k}^{N / 2} \exp \left[-\frac{\beta_{k}}{2}\left\|\mathbf{y}_{k}-\mathbf{B}_{k}\left(\mathbf{s}_{k}\right) \mathbf{x}\right\|^{2}\right] .
$$

Assuming statistical independence of the noise among the LR image acquisitions, the conditional probability of the set of LR images $\mathbf{y}$ given $\mathbf{x}$ can be expressed as

$$
\begin{aligned}
\mathrm{p}\left(\mathbf{y} \mid \mathbf{x},\left\{\mathbf{s}_{k}\right\},\left\{\beta_{k}\right\}\right) \\
=\prod_{k=1}^{L} \mathrm{p}\left(\mathbf{y}_{k} \mid \mathbf{x}, \mathbf{s}_{k}, \beta_{k}\right) \\
\propto\left[\prod_{k=1}^{L} \beta_{k}^{N / 2}\right] \exp \left[-\frac{1}{2} \sum_{k=1}^{L} \beta_{k}\left\|\mathbf{y}_{k}-\mathbf{B}_{k}\left(\mathbf{s}_{k}\right) \mathbf{x}\right\|^{2}\right] .
\end{aligned}
$$

The independent Gaussian model in (3) is used in most of the existing super resolution methods [8], [9], [15]-[17], [28]. Some methods utilized $l_{1}$-norm based observation models which take both acquisition and registration noise into account [5], [6]. In this paper, we use (3) to model only the acquisition noise. We incorporate an explicit modeling of the registration errors separately and therefore they are not taken into account in (3).

Let us now explicitly state the form of the matrices $\mathbf{C}\left(\mathbf{s}_{k}\right)$. We denote the coordinates of the reference HR grid by $(u, v)$ and the coordinates of the $k$ th warped HR grid, after applying $\mathbf{C}\left(\mathbf{s}_{k}\right)$ to $\mathbf{x}$, by $\left(u_{k}, v_{k}\right)$. Let us also define

$$
\begin{aligned}
& \Delta u_{k}=u_{k}-u=u \cos \left(\theta_{k}\right)-v \sin \left(\theta_{k}\right)+c_{k}-u \\
& \Delta v_{k}=v_{k}-v=u \sin \left(\theta_{k}\right)+v \cos \left(\theta_{k}\right)+d_{k}-v .
\end{aligned}
$$

Note that the coordinates $\left(u_{k}, v_{k}\right)$ generally correspond to fractional values, and therefore the HR image value at pixel $\left(u_{k}, v_{k}\right)$ in the $k$ th HR grid has to be calculated using resampling. As in [21], we incorporate bilinear interpolation to approximate the HR image value at $\left(u_{k}, v_{k}\right)$ using the four neighboring HR image values $x_{t l\left(\mathbf{s}_{k}\right)}, x_{t r\left(\mathbf{s}_{k}\right)}, x_{b l\left(\mathbf{s}_{k}\right)}$, and $x_{b r\left(\mathbf{s}_{k}\right)}$, which are the pixels at the top-left, top-right, bottom-left and bottom-right locations of the pixel at $\left(u_{k}, v_{k}\right)$, respectively.

Let us denote by $\left(a_{k}\left(\mathbf{s}_{k}\right), b_{k}\left(\mathbf{s}_{k}\right)\right)^{T}$ the vector difference between the pixel at $\left(u_{k}, v_{k}\right)$ and the pixel at its top-left position in the reference HR grid, that is

$$
\begin{aligned}
& a_{k}\left(\mathbf{s}_{k}\right)=\Delta u_{k}-\text { floor }\left(\Delta u_{k}\right) \\
& b_{k}\left(\mathbf{s}_{k}\right)=\Delta v_{k}-\text { floor }\left(\Delta v_{k}\right) .
\end{aligned}
$$

Using bilinear interpolation, the warped image $\mathbf{C}\left(\mathbf{s}_{k}\right) \mathbf{x}$ can be approximated as (see [21] for details)

$$
\begin{aligned}
\mathbf{C}\left(\mathbf{s}_{k}\right) \mathbf{x} \approx & \mathbf{D}_{\mathbf{b}_{k}\left(\mathbf{s}_{k}\right)}\left(\mathbf{I}-\mathbf{D}_{\mathbf{a}_{k}\left(\mathbf{s}_{k}\right)}\right) \mathbf{L}_{\mathbf{b l}\left(\mathbf{s}_{k}\right)} \mathbf{x} \\
& +\mathbf{D}_{\mathbf{b}_{k}\left(\mathbf{s}_{k}\right)} \mathbf{D}_{\mathbf{a}_{k}\left(\mathbf{s}_{k}\right)} \mathbf{L}_{\mathbf{b r}\left(\mathbf{s}_{k}\right)} \mathbf{x} \\
& +\left(\mathbf{I}-\mathbf{D}_{\mathbf{b}_{k}\left(\mathbf{s}_{k}\right)}\right)\left(\mathbf{I}-\mathbf{D}_{\mathbf{a}_{k}\left(\mathbf{s}_{k}\right)}\right) \mathbf{L}_{\mathbf{t l}\left(\mathbf{s}_{k}\right)} \mathbf{x} \\
& +\left(\mathbf{I}-\mathbf{D}_{\mathbf{b}_{k}\left(\mathbf{s}_{k}\right)}\right) \mathbf{D}_{\mathbf{a}_{k}\left(\mathbf{s}_{k}\right)} \mathbf{L}_{\mathbf{t r}\left(\mathbf{s}_{k}\right)} \mathbf{x}
\end{aligned}
$$

where $\mathbf{D}_{\mathbf{a}_{k}\left(\mathbf{s}_{k}\right)}$ and $\mathbf{D}_{\mathbf{b}_{k}\left(\mathbf{s}_{k}\right)}$ denote diagonal matrices with the vectors $\mathbf{a}_{k}\left(\mathbf{s}_{k}\right)$ and $\mathbf{b}_{k}\left(\mathbf{s}_{k}\right)$ in their diagonal, respectively. The matrices $\mathbf{L}_{\mathbf{z}}$ with $\mathbf{z} \in\left\{\mathbf{b l}\left(\mathbf{s}_{k}\right), \mathbf{b r}\left(\mathbf{s}_{k}\right), \mathbf{t l}\left(\mathbf{s}_{k}\right), \operatorname{tr}\left(\mathbf{s}_{k}\right)\right\}$ are constructed in such a way that the product $\mathbf{L}_{\mathbf{z}} \mathbf{x}$ produces pixels at the top-left, top-right, bottom-left and bottom-right locations of $\left(u_{k}, v_{k}\right)$, respectively.

\section{B. Image Model}

The quality of the estimated HR image as well as the accuracy in the estimates of other unknowns depends on the incorporation of accurate image models. The TV function is utilized successfully in a number of image recovery methods including denoising [29], blind deconvolution [30], inpainting, and super resolution [31]. TV priors are very effective in preserving edges while imposing smoothness. In this work, as the HR image prior, we utilize the quadratic approximation of the TV prior, that is

$$
\mathrm{p}\left(\mathbf{x} \mid \alpha_{\mathrm{im}}\right)=c \alpha_{\mathrm{im}}^{P N / 2} \exp \left[-\frac{1}{2} \alpha_{\mathrm{im}} \mathrm{TV}(\mathbf{x})\right]
$$

where $c$ is a constant and

$$
\mathrm{TV}(\mathbf{x})=\sum_{i=1}^{P N} \sqrt{\left(\Delta_{i}^{h}(\mathbf{x})\right)^{2}+\left(\Delta_{i}^{v}(\mathbf{x})\right)^{2}}
$$

The operators $\Delta_{i}^{h}(\mathbf{x})$ and $\Delta_{i}^{v}(\mathbf{x})$ correspond, respectively, to the horizontal and vertical first order differences at pixel $i$. Let us also define the horizontal and vertical first order difference matrices $\Delta^{h}$ and $\Delta^{v}$, such that $\left(\Delta^{h} \mathbf{x}\right)_{i}=\Delta_{i}^{h}(\mathbf{x})$ and $\left(\Delta^{v} \mathbf{x}\right)_{i}=$ $\Delta_{i}^{v}(\mathbf{x})$.

\section{Modeling the Uncertainties in the Registration Parameters}

Let us denote by $\overline{\mathbf{s}}_{k}^{p}$ the estimate of $\mathbf{s}_{k}$ obtained from LR observations in a preprocessing step, using registration algorithms, such as the ones reported in [32], [33]. As mentioned earlier, these estimates are in general inaccurate, which lowers the image restoration quality. Therefore, we model the motion parameters as stochastic variables following Gaussian distributions with a priori means set equal to the preliminary motion parameters $\overline{\mathbf{s}}_{k}^{p}$, that is

$$
\mathrm{p}\left(\mathbf{s}_{k}\right)=\mathcal{N}\left(\mathbf{s}_{k} \mid \overline{\mathbf{s}}_{k}^{p}, \boldsymbol{\Lambda}_{k}^{p}\right)
$$

with $\boldsymbol{\Lambda}_{k}^{p}$ the a priori covariance matrix. The parameters $\overline{\mathbf{s}}_{k}^{p}$ and $\boldsymbol{\Lambda}_{k}^{p}$ incorporate prior knowledge about the motion parameters into the estimation procedure. If such knowledge is not available, $\overline{\mathbf{s}}_{k}^{p}$ and $\left(\boldsymbol{\Lambda}_{k}^{p}\right)^{-1}$ can be set equal to zero, which makes the observations solely responsible for the estimation process. Similar models utilizing Gaussian distributions to model the uncertainty in preliminary motion parameters have also been used in some existing algorithms [8], [16], [17], but with different inference methods.

\section{Hyperpriors on the Hyperparameters}

The hyperparameters $\alpha_{\mathrm{im}}$ and $\left\{\beta_{k}\right\}$ are crucial in determining the performance of the SR algorithm. For their modeling, we employ Gamma distributions

$$
\mathrm{p}(\omega)=\Gamma\left(\omega \mid a_{\omega}^{o}, b_{\omega}^{o}\right)=\frac{\left(b_{\omega}^{o}\right)^{a_{\omega}^{o}}}{\Gamma\left(a_{\omega}^{o}\right)} \omega^{a_{\omega}^{o}-1} \exp \left[-b_{\omega}^{o} \omega\right]
$$


where $\omega>0$ denotes a hyperparameter, and $a_{\omega}^{o}>0$ and $b_{\omega}^{o}>0$ are the shape and scale parameters, respectively. The hyperpriors are chosen as Gamma distributions since they are the conjugate priors for the variance of the Gaussian distribution, that is, they have the same functional form with the product of the prior distributions and the observation model [34].

Finally, combining (3), (9), (11) and (12), we obtain the joint probability distribution of all variables as

$$
\begin{aligned}
\mathrm{p}\left(\mathbf{y}, \mathbf{x},\left\{\mathbf{s}_{k}\right\}, \alpha_{\mathrm{im}},\right. & \left.\left\{\beta_{k}\right\}\right)=\mathrm{p}\left(\mathbf{y} \mid \mathbf{x},\left\{\mathbf{s}_{k}\right\},\left\{\beta_{k}\right\}\right) \\
& \times \mathrm{p}\left(\mathbf{x} \mid \alpha_{\mathrm{im}}\right) \mathrm{p}\left(\mathbf{s}_{k}\right) \mathrm{p}\left(\alpha_{\mathrm{im}}\right) \prod_{k=1}^{L} \mathrm{p}\left(\beta_{k}\right) .
\end{aligned}
$$

\section{VARIATIONAL BAYESIAN INFERENCE}

Let us first denote the set of all unknowns by $\Theta=$ $\left\{\mathbf{x},\left\{\mathbf{s}_{k}\right\}, \alpha_{\mathrm{im}},\left\{\beta_{k}\right\}\right\}$ for clarity. The Bayesian inference is based on the posterior distribution

$$
p(\Theta \mid \mathbf{y})=\frac{p(\Theta, \mathbf{y})}{p(\mathbf{y})} .
$$

However, as in many applications, this distribution is intractable, since $p(y)$ cannot be computed. Therefore, approximation methods are utilized, some of which are evidence analysis (type-II maximum likelihood) and sampling methods [35]. In this work, we resort to a variational Bayesian analysis due to its certain advantages, including accounting for the uncertainties in the estimation processes and computational efficiency compared to the sampling approaches, among others.

In the variational Bayesian analysis, the posterior distribution $p(\Theta \mid \mathbf{y})$ is approximated by a tractable distribution $q(\Theta)$. This approximating distribution is found by minimizing the Kullback-Leibler (KL) distance between $\mathrm{q}(\Theta)$ and the posterior $\mathrm{p}(\Theta \mid \mathbf{y})$, given by

$$
\begin{aligned}
& C_{K L}(\mathrm{q}(\Theta) \| \mathrm{p}(\Theta \mid \mathbf{y})) \\
& \quad=\int \mathrm{q}(\Theta) \log \left(\frac{\mathrm{q}(\Theta)}{\mathrm{p}(\Theta \mid \mathbf{y})}\right) d \Theta \\
& \quad=\int \mathrm{q}(\Theta) \log \left(\frac{\mathrm{q}(\Theta)}{\mathrm{p}(\Theta, \mathbf{y})}\right) d \Theta+\text { const. }
\end{aligned}
$$

Generally, the only assumption made in variational Bayesian analysis is that the distribution $\mathrm{q}(\Theta)$ can be factorized [35]-[37]. In this work, we use the following factorization

$$
\begin{aligned}
\mathrm{q}(\Theta) & =\mathrm{q}\left(\mathbf{x},\left\{\mathbf{s}_{k}\right\}, \alpha_{\mathrm{im}},\left\{\beta_{k}\right\}\right) \\
& =\mathrm{q}\left(\alpha_{\mathrm{im}}\right) \mathrm{q}(\mathbf{x}) \prod_{k=1}^{L} \mathrm{q}\left(\mathbf{s}_{k}\right) \prod_{k=1}^{L} \mathrm{q}\left(\beta_{k}\right) .
\end{aligned}
$$

Unfortunately, we can not directly calculate the KL distance because of the TV image prior. In earlier work with TV priors, this difficulty is overcome by resorting to majorization-minimization (MM) approaches, which is also the method adopted in this paper. In the following we present an outline of the MM approach (details can be found in [38]).
The main principle of the MM approach is to find a bound of the joint distribution in (13) which makes the minimization of (15) tractable. A lower bound of the distribution in (13) can be found as follows. Let us first consider the following inequality, derived from the geometric-arithmetic mean inequality, which states that for real numbers $a \geq 0$ and $b>0$

$$
\sqrt{a b} \leq \frac{a+b}{2} \Rightarrow \sqrt{a} \leq \frac{a+b}{2 \sqrt{b}} .
$$

Next we define the functional $\mathrm{M}\left(\alpha_{\mathrm{im}}, \mathbf{x}, \mathbf{w}\right)$ with a $P N$-dimensional vector $\mathbf{w} \in\left(R^{+}\right)^{P N}$, with components $w_{i}, \quad i=$ $1, \ldots, P N$, as follows:

$$
\begin{aligned}
& \mathrm{M}\left(\alpha_{\mathrm{im}}, \mathbf{x}, \mathbf{w}\right)=c \alpha_{\mathrm{im}}^{P N / 2} \\
& \quad \times \exp \left[-\frac{\alpha_{\mathrm{im}}}{2} \sum_{i} \frac{\left(\Delta_{i}^{h}(\mathbf{x})\right)^{2}+\left(\Delta_{i}^{v}(\mathbf{x})\right)^{2}+w_{i}}{\sqrt{w_{i}}}\right] .
\end{aligned}
$$

As will be clear later, the auxiliary variable $\mathrm{w}$ is a quantity that needs to be computed and it has an interpretation related to the unknown HR image $\mathbf{x}$. Using $a=\left(\Delta_{i}^{h}(\mathbf{x})\right)^{2}+\left(\Delta_{i}^{v}(\mathbf{x})\right)^{2}$ and $b=w_{i}$ in the inequality (17) it is easy to show that the functional $\mathrm{M}\left(\alpha_{\mathrm{im}}, \mathbf{x}, \mathbf{w}\right)$ is a lower bound of the image prior $\mathrm{p}\left(\mathbf{x} \mid \alpha_{\mathrm{im}}\right)$, that is

$$
\mathrm{p}\left(\mathbf{x} \mid \alpha_{\mathrm{im}}\right) \geq \mathrm{M}\left(\alpha_{\mathrm{im}}, \mathbf{x}, \mathbf{w}\right) .
$$

This lower bound can be used to find a lower bound for the joint distribution in (13)

$$
\begin{aligned}
\mathrm{p}(\mathbf{y}, \Theta) & \geq \mathrm{p}(\mathbf{y} \mid \Theta) \mathrm{M}\left(\alpha_{\mathrm{im}}, \mathbf{x}, \mathbf{w}\right) \mathrm{p}\left(\mathbf{s}_{k}\right) \mathrm{p}\left(\alpha_{\mathrm{im}}\right) \prod_{k=1}^{L} \mathrm{p}\left(\beta_{k}\right) \\
& =\mathrm{F}(\Theta, \mathbf{w}, \mathbf{y}),
\end{aligned}
$$

which results in an upper bound of the KL distance in (15) as

$$
C_{K L}(\mathrm{q}(\Theta) \| \mathrm{p}(\Theta, \mathbf{y})) \leq C_{K L}(\mathrm{q}(\Theta) \| \mathrm{F}(\Theta, \mathbf{w}, \mathbf{y})) .
$$

It has been shown in [30] that the minimization of (15) can be replaced by the minimization of its upper bound (21), as minimizing this bound with respect to the unknowns and the auxiliary variable $\mathbf{w}$ in an alternating fashion results in closer bounds at each iteration. The bound in (21) is quadratic and therefore it is easy to evaluate analytically. The standard solutions of the variational Bayesian methods [35] can then be used by replacing $\mathrm{p}(\mathbf{y}, \Theta)$ with $\mathrm{F}(\Theta, \mathbf{w}, \mathbf{y})$ to estimate the unknown distributions $\mathrm{q}(\xi)$ with $\xi \in \Theta$ as follows

$$
\mathrm{q}(\xi)=\text { const } \times \exp \left(\langle\log \mathrm{F}(\Theta, \mathbf{w}, \mathbf{y})\rangle_{\mathrm{q}\left(\Theta_{\xi}\right)}\right)
$$

where $\Theta_{\xi}$ denotes the set $\Theta$ with $\xi$ removed and $\mathrm{E}_{\Theta_{\xi}}[\cdot]=$ $\langle\cdot\rangle_{q\left(\Theta_{\xi}\right)}$ denotes expected value with respect to the distribution $\mathrm{q}\left(\Theta_{\xi}\right)$. In the following, the subscript of the expected value will be removed when it is clear from the context.

Let us now proceed with deriving the explicit forms of the solutions for each unknown using (22). 


\section{A. Estimation of the HR Image Distribution}

From (22), the distribution $\mathrm{q}(\mathbf{x})$ can be found as

$$
\begin{aligned}
\mathrm{q}(\mathbf{x}) \propto \exp & \left(-\frac{1}{2}\left\langle\alpha_{\mathrm{im}}\right\rangle \sum_{i} \frac{\left(\Delta_{i}^{h}(\mathbf{x})\right)^{2}+\left(\Delta_{i}^{v}(\mathbf{x})\right)^{2}}{\sqrt{w_{i}}}\right. \\
& \left.-\frac{1}{2} \sum_{k}\left\langle\beta_{k}\right\rangle \mathrm{E}_{\mathbf{s}_{k}}\left[\left\|\mathbf{y}_{k}-\mathbf{A} \mathbf{H}_{k} \mathbf{C}\left(\mathbf{s}_{k}\right) \mathbf{x}\right\|^{2}\right]\right) .
\end{aligned}
$$

The explicit form of this distribution depends on the expectation $\mathrm{E}_{\mathbf{s}_{k}}\left[\left\|\mathbf{y}_{k}-\mathbf{A H}_{k} \mathbf{C}\left(\mathbf{s}_{k}\right) \mathbf{x}\right\|^{2}\right]$. This calculation is not easy since $\mathbf{C}\left(\mathbf{s}_{k}\right) \mathbf{x}$ is nonlinear with respect to $\mathbf{s}_{k}$. Therefore, we expand $\mathbf{C}\left(\mathbf{s}_{k}\right) \mathbf{x}$ using its first-order Taylor series around the mean value $\left\langle\mathbf{s}_{k}\right\rangle=\overline{\mathbf{s}}_{k}=\left(\bar{\theta}_{k}, \bar{c}_{k}, \bar{d}_{k}\right)^{T}$ of the distribution $\mathrm{q}\left(\mathbf{s}_{k}\right)$. Proceeding in this fashion, we obtain the following approximation of $\mathbf{C}\left(\mathbf{s}_{k}\right) \mathbf{x}$

$$
\mathbf{C}\left(\mathbf{s}_{k}\right) \mathbf{x} \approx \mathbf{C}\left(\overline{\mathbf{s}}_{k}\right) \mathbf{x}+\mathbf{L}_{\mathbf{x}}\left(\overline{\mathbf{s}}_{k}\right) \mathbf{P}\left(\overline{\mathbf{s}}_{k}\right)\left(\mathbf{s}_{k}-\overline{\mathbf{s}}_{k}\right)
$$

where

$$
\begin{aligned}
\mathbf{L}_{\mathbf{x}}\left(\overline{\mathbf{s}}_{k}\right)= & {\left[\operatorname { d i a g } \left\{\left(\mathbf{I}-\mathbf{D}_{\mathbf{b}_{k}\left(\mathbf{s}_{k}\right)}\right)\left(\mathbf{L}_{\mathbf{t r}\left(\mathbf{s}_{k}\right)}-\mathbf{L}_{\mathbf{t l}\left(\mathbf{s}_{k}\right)}\right) \mathbf{x}\right.\right.} \\
& \left.+\mathbf{D}_{\mathbf{b}_{k}\left(\mathbf{s}_{k}\right)}\left(\mathbf{L}_{\mathbf{b r}\left(\mathbf{s}_{k}\right)}-\mathbf{L}_{\mathbf{b l}\left(\mathbf{s}_{k}\right)}\right) \mathbf{x}\right\} \\
& \operatorname{diag}\left\{\left(\mathbf{I}-\mathbf{D}_{\mathbf{a}_{k}\left(\mathbf{s}_{k}\right)}\right)\left(\mathbf{L}_{\mathbf{b l}\left(\mathbf{s}_{k}\right)}-\mathbf{L}_{\mathbf{t l}\left(\mathbf{s}_{k}\right)}\right) \mathbf{x}\right. \\
& \left.\left.+\mathbf{D}_{\mathbf{a}_{k}\left(\mathbf{s}_{k}\right)}\left(\mathbf{L}_{\mathbf{b r}\left(\mathbf{s}_{k}\right)}-\mathbf{L}_{\mathbf{t r}\left(\mathbf{s}_{k}\right)}\right) \mathbf{x}\right\}\right]
\end{aligned}
$$

and

$$
\mathbf{P}\left(\overline{\mathbf{s}}_{k}\right)=\left(\begin{array}{ccc}
-\mathbf{u} \sin \left(\bar{\theta}_{k}\right)-\mathbf{v} \cos \left(\bar{\theta}_{k}\right) & \mathbf{1} & \mathbf{0} \\
\mathbf{u} \cos \left(\bar{\theta}_{k}\right)-\mathbf{v} \sin \left(\bar{\theta}_{k}\right) & \mathbf{0} & \mathbf{1}
\end{array}\right),
$$

where $\mathbf{u}$ and $\mathbf{v}$ are vectors constructed by lexicographically arranging the coordinates of the reference HR grid. We now rewrite $\mathbf{L}_{\mathbf{x}}\left(\overline{\mathbf{s}}_{k}\right) \mathbf{P}\left(\overline{\mathbf{s}}_{k}\right)$ in a more convenient form that allows for square completion with respect to $\mathbf{x}$ in (23). We first define the matrices

$$
\begin{aligned}
\mathbf{M}_{1}\left(\overline{\mathbf{s}}_{k}\right)= & \left(\mathbf{I}-\mathbf{D}_{\mathbf{b}_{k}\left(\mathbf{s}_{k}\right)}\right)\left(\mathbf{L}_{\mathbf{t r}\left(\mathbf{s}_{k}\right)}-\mathbf{L}_{\mathbf{t l}\left(\mathbf{s}_{k}\right)}\right) \\
& +\mathbf{D}_{\mathbf{b}_{k}\left(\mathbf{s}_{k}\right)}\left(\mathbf{L}_{\mathbf{b r}\left(\mathbf{s}_{k}\right)}-\mathbf{L}_{\mathbf{b l}\left(\mathbf{s}_{k}\right)}\right) \\
\mathbf{M}_{2}\left(\overline{\mathbf{s}}_{k}\right)= & \left(\mathbf{I}-\mathbf{D}_{\mathbf{a}_{k}\left(\mathbf{s}_{k}\right)}\right)\left(\mathbf{L}_{\mathbf{b l}\left(\mathbf{s}_{k}\right)}-\mathbf{L}_{\mathbf{t l}\left(\mathbf{s}_{k}\right)}\right) \\
& +\mathbf{D}_{\mathbf{a}_{k}\left(\mathbf{s}_{k}\right)}\left(\mathbf{L}_{\mathbf{b r}\left(\mathbf{s}_{k}\right)}-\mathbf{L}_{\mathbf{t r}\left(\mathbf{s}_{k}\right)}\right),
\end{aligned}
$$

and

$$
\begin{aligned}
& \mathbf{P}_{1}\left(\overline{\mathbf{s}}_{k}\right)=\operatorname{diag}\left(-\mathbf{u} \sin \left(\bar{\theta}_{k}\right)-\mathbf{v} \cos \left(\bar{\theta}_{k}\right)\right) \\
& \mathbf{P}_{2}\left(\overline{\mathbf{s}}_{k}\right)=\operatorname{diag}\left(\mathbf{u} \cos \left(\bar{\theta}_{k}\right)-\mathbf{v} \sin \left(\bar{\theta}_{k}\right)\right) .
\end{aligned}
$$

Then, $\mathbf{L}_{\mathbf{x}}\left(\overline{\mathbf{s}}_{k}\right) \mathbf{P}\left(\overline{\mathbf{s}}_{k}\right)$ can expressed as

$$
\begin{aligned}
\mathbf{L}_{\mathbf{x}}\left(\bar{s}_{k}\right) \mathbf{P}\left(\bar{s}_{k}\right)= & {\left[\left(\mathbf{P}_{1}\left(\overline{\mathbf{s}}_{k}\right) \mathbf{M}_{1}\left(\overline{\mathbf{s}}_{k}\right)+\mathbf{P}_{2}\left(\overline{\mathbf{s}}_{k}\right)\right.\right.} \\
& \left.\left.\times \mathbf{M}_{2}\left(\overline{\mathbf{s}}_{k}\right)\right) \mathbf{x}, \mathbf{M}_{1}\left(\overline{\mathbf{s}}_{k}\right) \mathbf{x}, \mathbf{M}_{2}\left(\overline{\mathbf{s}}_{k}\right) \mathbf{x}\right] \\
= & {\left[\mathbf{N}_{1}\left(\overline{\mathbf{s}}_{k}\right) \mathbf{x}, \mathbf{N}_{2}\left(\overline{\mathbf{s}}_{k}\right) \mathbf{x}, \mathbf{N}_{3}\left(\overline{\mathbf{s}}_{k}\right) \mathbf{x}\right] }
\end{aligned}
$$

such that using (24) we obtain

$$
\begin{aligned}
\mathbf{B}\left(\mathbf{s}_{k}\right) \mathbf{x}= & \mathbf{A H}_{k} \mathbf{C}\left(\mathbf{s}_{k}\right) \mathbf{x} \\
\approx & \mathbf{A H} \mathbf{H}_{k} \mathbf{C}\left(\overline{\mathbf{s}}_{k}\right) \mathbf{x}+\mathbf{A} \mathbf{H}_{k} \\
& \times\left[\mathbf{N}_{1}\left(\overline{\mathbf{s}}_{k}\right) \mathbf{x}, \mathbf{N}_{2}\left(\overline{\mathbf{s}}_{k}\right) \mathbf{x}, \mathbf{N}_{3}\left(\overline{\mathbf{s}}_{k}\right) \mathbf{x}\right]\left(\mathbf{s}_{k}-\overline{\mathbf{s}}_{k}\right) \\
= & \mathbf{A} \mathbf{H}_{k} \mathbf{C}\left(\overline{\mathbf{s}}_{k}\right) \mathbf{x} \\
& +\left[\mathbf{O}_{k 1}\left(\overline{\mathbf{s}}_{k}\right) \mathbf{x}, \mathbf{O}_{k 2}\left(\overline{\mathbf{s}}_{k}\right) \mathbf{x}, \mathbf{O}_{k 3}\left(\overline{\mathbf{s}}_{k}\right) \mathbf{x}\right]\left(\mathbf{s}_{k}-\overline{\mathbf{s}}_{k}\right)
\end{aligned}
$$

with

$$
\mathbf{O}_{k r}\left(\overline{\mathbf{s}}_{k}\right)=\mathbf{A} \mathbf{H}_{k} \mathbf{N}_{r}\left(\overline{\mathbf{s}}_{k}\right), \quad r=1,2,3 .
$$

Notice that instead of the first-order expansion in (24), a second-order expansion can be employed on the error term in (23) (similar to [17]). The quantity $\sum_{k}\left\langle\beta_{k}\right\rangle \mathrm{E}_{\mathbf{s}_{k}}\left[\left\|\mathbf{y}_{k}-\mathbf{A H}_{k} \mathbf{C}\left(\mathbf{s}_{k}\right) \mathbf{x}\right\|^{2}\right]$ can then be calculated using (33) as

$$
\begin{aligned}
& \sum_{k}\left\langle\beta_{k}\right\rangle \mathrm{E}_{\mathbf{s}_{k}}\left[\left\|\mathbf{y}_{k}-\mathbf{A} \mathbf{H}_{k} \mathbf{C}\left(\mathbf{s}_{k}\right) \mathbf{x}\right\|^{2}\right] \\
& \approx \sum_{k}\left\langle\beta_{k}\right\rangle\left\|\mathbf{y}_{k}-\mathbf{A} \mathbf{H}_{k} \mathbf{C}\left(\overline{\mathbf{s}}_{k}\right) \mathbf{x}\right\|^{2} \\
&+\sum_{k}\left\langle\beta_{k}\right\rangle \operatorname{trace}\left(\left[\mathbf{O}_{k 1}\left(\overline{\mathbf{s}}_{k}\right) \mathbf{x}, \mathbf{O}_{k 2}\left(\overline{\mathbf{s}}_{k}\right) \mathbf{x}, \mathbf{O}_{k 3}\left(\overline{\mathbf{s}}_{k}\right) \mathbf{x}\right]^{T}\right. \\
&\left.\times\left[\mathbf{O}_{k 1}\left(\overline{\mathbf{s}}_{k}\right) \mathbf{x}, \mathbf{O}_{k 2}\left(\overline{\mathbf{s}}_{k}\right) \mathbf{x}, \mathbf{O}_{k 3}\left(\overline{\mathbf{s}}_{k}\right) \mathbf{x}\right] \mathbf{\Lambda}_{k}\right) \\
&= \sum_{k}\left\langle\beta_{k}\right\rangle\left\|\mathbf{y}_{k}-\mathbf{B}\left(\overline{\mathbf{s}}_{k}\right) \mathbf{x}\right\|^{2} \\
&+\sum_{k} \sum_{i=1}^{3} \sum_{j=1}^{3}\left\langle\beta_{k}\right\rangle \lambda_{k i j} \mathbf{x}^{T} \mathbf{O}_{k i}\left(\overline{\mathbf{s}}_{k}\right)^{T} \mathbf{O}_{k j}\left(\overline{\mathbf{s}}_{k}\right) \mathbf{x}
\end{aligned}
$$

where $\boldsymbol{\Lambda}_{k}$ is the covariance matrix of the posterior distribution $\mathrm{q}\left(\mathbf{s}_{k}\right)$ constructed with elements $\lambda_{k i j}, i=1,2,3, j=1,2,3$, that is

$$
\boldsymbol{\Lambda}_{k}=\left(\begin{array}{lll}
\lambda_{k 11} & \lambda_{k 12} & \lambda_{k 13} \\
\lambda_{k 21} & \lambda_{k 22} & \lambda_{k 23} \\
\lambda_{k 31} & \lambda_{k 32} & \lambda_{k 33}
\end{array}\right)
$$

Finally, using (35) in (23), the posterior distribution $q(\mathbf{x})$ of the HR image $\mathbf{x}$ is found to be a multivariate Gaussian distribution given by

$$
\mathrm{q}(\mathbf{x})=\mathcal{N}\left(\mathbf{x} \mid \mu_{\mathbf{x}}, \Sigma_{\mathbf{x}}\right)
$$

with parameters

$$
\begin{aligned}
\mu_{\mathbf{x}}= & \Sigma_{\mathbf{x}}\left[\sum_{k}\left\langle\beta_{k}\right\rangle \mathbf{B}_{k}\left(\overline{\mathbf{s}}_{k}\right)^{T} \mathbf{y}_{k}\right] \\
\Sigma_{\mathbf{x}}^{-1}= & \sum_{k}\left\langle\beta_{k}\right\rangle \mathbf{B}_{k}\left(\overline{\mathbf{s}}_{k}\right)^{T} \mathbf{B}_{k}\left(\overline{\mathbf{s}}_{k}\right) \\
& +\sum_{k} \sum_{i=1}^{3} \sum_{j=1}^{3}\left\langle\beta_{k}\right\rangle \lambda_{k i j} \mathbf{O}_{k i}\left(\overline{\mathbf{s}}_{k}\right)^{T} \mathbf{O}_{k j}\left(\overline{\mathbf{s}}_{k}\right) \\
& +\left\langle\alpha_{\mathrm{im}}\right\rangle\left(\Delta^{h}\right)^{T} \mathbf{W} \Delta^{h}+\left\langle\alpha_{\mathrm{im}}\right\rangle\left(\Delta^{v}\right)^{T} \mathbf{W} \Delta^{v},
\end{aligned}
$$

where

$$
\mathbf{W}=\operatorname{diag}\left(\frac{1}{\sqrt{w_{i}}}\right), \quad i=1, \ldots, P N .
$$

The elements $w_{i}$ of the auxiliary vector $\mathbf{w}=$ $\left(w_{1}, w_{2}, \ldots, w_{P N}\right)$ are calculated by minimizing the upper bound in (21), which results in

$$
\begin{aligned}
w_{i}= & \mathrm{E}_{\mathbf{x}}\left[\left(\Delta_{i}^{h}(\mathbf{x})\right)^{2}+\left(\Delta_{i}^{v}(\mathbf{x})\right)^{2}\right] \\
= & \left(\Delta_{i}^{h} \mu_{\mathbf{x}}\right)^{2}+\left(\Delta_{i}^{v} \mu_{\mathbf{x}}\right)^{2}+\operatorname{trace}\left[\left(\Delta_{i}^{h}\right)^{T}\left(\Delta_{i}^{h}\right) \Sigma_{\mathbf{x}}\right] \\
& +\operatorname{trace}\left[\left(\Delta_{i}^{v}\right)^{T}\left(\Delta_{i}^{v}\right) \Sigma_{\mathbf{x}}\right], \quad i=1, \ldots, P N,
\end{aligned}
$$


where $\Delta_{i}^{h}$ and $\Delta_{i}^{v}$ represent respectively the horizontal and vertical difference matrices at pixel $i$. It is clear from (40) that the vector $\mathbf{w}$ represents the local spatial activity in the HR image $\mathbf{x}$. Therefore, the matrix $\mathbf{W}$ introduces spatial adaptivity into the estimation process of the HR image in (37)-(38) by controlling the smoothing applied at different locations. Moreover, the uncertainty of the image estimate is also taken into account by the last two terms in (40) when calculating the spatial adaptivity vector $\mathbf{w}$ using the distribution $q(\mathbf{x})$ (see also [38] for a related discussion).

\section{B. Estimation of the Registration Parameter Distributions}

The posterior distribution approximation $q\left(\mathbf{s}_{k}\right)$ is found from (22) as

$$
\begin{aligned}
\mathrm{q}\left(\mathbf{s}_{k}\right) \propto \exp \left(-\frac{1}{2}\left\langle\beta_{k}\right\rangle \mathrm{E}_{\mathbf{x}}\left[\left\|\mathbf{y}_{k}-\mathbf{B}_{k}\left(\mathbf{s}_{k}\right) \mathbf{x}\right\|^{2}\right]\right. \\
\left.\quad-\frac{1}{2}\left(\mathbf{s}_{k}-\overline{\mathbf{s}}_{k}^{p}\right)^{T}\left(\boldsymbol{\Lambda}_{k}^{p}\right)^{-1}\left(\mathbf{s}_{k}-\overline{\mathbf{s}}_{k}^{p}\right)\right) .
\end{aligned}
$$

To obtain the explicit form of this distribution, the expectation $\mathrm{E}_{\mathbf{x}}\left[\left\|\mathbf{y}_{k}-\mathbf{B}_{k}\left(\mathbf{s}_{k}\right) \mathbf{x}\right\|^{2}\right]$ needs to be calculated. We proceed as in the previous section by using its Taylor series expansion around $\overline{\mathbf{s}}_{k}$, the estimate of the registration parameters obtained in the previous iteration. By utilizing the approximation (33) to obtain

$$
\begin{aligned}
\mathrm{E}_{\mathbf{x}}[ & \left.\left\|\mathbf{y}_{k}-\mathbf{B}_{k}\left(\mathbf{s}_{k}\right) \mathbf{x}\right\|^{2}\right] \\
= & \mathrm{E}_{\mathbf{x}}\left[\| \mathbf{y}_{k}-\mathbf{B}\left(\overline{\mathbf{s}}_{k}\right) \mathbf{x}\right. \\
& \left.-\left[\mathbf{O}_{k 1}\left(\overline{\mathbf{s}}_{k}\right) \mathbf{x}, \mathbf{O}_{k 1}\left(\overline{\mathbf{s}}_{k}\right) \mathbf{x}, \mathbf{O}_{k 1}\left(\overline{\mathbf{s}}_{k}\right) \mathbf{x}\right]\left(\mathbf{s}_{k}-\overline{\mathbf{s}}_{k}\right) \|^{2}\right] \\
= & \| \mathbf{y}_{k}-\mathbf{B}\left(\overline{\mathbf{s}}_{k}\right) \mu_{\mathbf{x}} \\
- & {\left[\mathbf{O}_{k 1}\left(\overline{\mathbf{s}}_{k}\right) \mu_{\mathbf{x}}, \mathbf{O}_{k 2}\left(\overline{\mathbf{s}}_{k}\right) \mu_{\mathbf{x}}, \mathbf{O}_{k 3}\left(\overline{\mathbf{s}}_{k}\right) \mu_{\mathbf{x}}\right]\left(\mathbf{s}_{k}-\overline{\mathbf{s}}_{k}\right) \|^{2} } \\
+ & \operatorname{trace}\left[\mathbf{B}\left(\overline{\mathbf{s}}_{k}\right)^{T} \mathbf{B}\left(\overline{\mathbf{s}}_{k}\right) \Sigma_{\mathbf{x}}\right] \\
+ & 2\left[\operatorname{trace}\left[\mathbf{B}\left(\overline{\mathbf{s}}_{k}\right)^{T} \mathbf{O}_{k 1}\left(\overline{\mathbf{s}}_{k}\right) \Sigma_{\mathbf{x}}\right]\right. \\
& \operatorname{trace}\left[\mathbf{B}\left(\overline{\mathbf{s}}_{k}\right)^{T} \mathbf{O}_{k 2}\left(\overline{\mathbf{s}}_{k}\right) \Sigma_{\mathbf{x}}\right] \\
& \left.\operatorname{trace}\left[\mathbf{B}\left(\overline{\mathbf{s}}_{k}\right)^{T} \mathbf{O}_{k 3}\left(\overline{\mathbf{s}}_{k}\right) \Sigma_{\mathbf{x}}\right]\right]\left(\mathbf{s}_{k}-\overline{\mathbf{s}}_{k}\right) \\
+ & \left(\mathbf{s}_{k}-\overline{\mathbf{s}}_{k}\right)^{T} \Psi_{k}\left(\mathbf{s}_{k}-\overline{\mathbf{s}}_{k}\right)
\end{aligned}
$$

with (43) shown at the bottom of the page. The distribution $q\left(\mathbf{s}_{k}\right)$ can then be explicitly expressed from (41) and (42) as a Gaussian distribution

$$
\mathrm{q}\left(\mathbf{s}_{k}\right)=\mathcal{N}\left(\mathbf{s}_{k} \mid\left\langle\mathbf{s}_{k}\right\rangle, \boldsymbol{\Lambda}_{k}\right)
$$

with parameters

$$
\begin{aligned}
\left\langle\mathbf{s}_{k}\right\rangle= & \boldsymbol{\Lambda}_{k}\left[\left(\boldsymbol{\Lambda}_{k}^{p}\right)^{-1} \overline{\mathbf{s}}_{k}^{p}+\left\langle\beta_{k}\right\rangle \Phi_{k} \overline{\mathbf{s}}_{k}+\left\langle\beta_{k}\right\rangle \Psi_{k} \overline{\mathbf{s}}_{k}\right. \\
+ & \left\langle\beta_{k}\right\rangle\left[\left(\mathbf{y}_{k}-\mathbf{B}\left(\overline{\mathbf{s}}_{k}\right) \mu_{\mathbf{x}}\right)^{T} \mathbf{O}_{k 1}\left(\overline{\mathbf{s}}_{k}\right) \mu_{\mathbf{x}}\right. \\
& \left(\mathbf{y}_{k}-\mathbf{B}\left(\overline{\mathbf{s}}_{k}\right) \mu_{\mathbf{x}}\right)^{T} \mathbf{O}_{k 2}\left(\overline{\mathbf{s}}_{k}\right) \mu_{\mathbf{x}} \\
& \left.\left(\mathbf{y}_{k}-\mathbf{B}\left(\overline{\mathbf{s}}_{k}\right) \mu_{\mathbf{x}}\right)^{T} \mathbf{O}_{k 3}\left(\overline{\mathbf{s}}_{k}\right) \mu_{\mathbf{x}}\right]^{T} \\
- & \left\langle\beta_{k}\right\rangle\left[\operatorname{trace}\left[\mathbf{B}\left(\overline{\mathbf{s}}_{k}\right)^{T} \mathbf{O}_{k 1}\left(\overline{\mathbf{s}}_{k}\right) \Sigma_{\mathbf{x}}\right]\right. \\
& \operatorname{trace}\left[\mathbf{B}\left(\overline{\mathbf{s}}_{k}\right)^{T} \mathbf{O}_{k 2}\left(\overline{\mathbf{s}}_{k}\right) \Sigma_{\mathbf{x}}\right] \\
& \left.\left.\operatorname{trace}\left[\mathbf{B}\left(\overline{\mathbf{s}}_{k}\right)^{T} \mathbf{O}_{k 3}\left(\overline{\mathbf{s}}_{k}\right) \Sigma_{\mathbf{x}}\right]\right]^{T}\right]
\end{aligned}
$$

and

$$
\boldsymbol{\Lambda}_{k}^{-1}=\left(\boldsymbol{\Lambda}_{k}^{p}\right)^{-1}+\left\langle\beta_{k}\right\rangle \Psi_{k}+\left\langle\beta_{k}\right\rangle \Phi_{k},
$$

with (47), shown at the bottom of the page

Note that the proposed registration method in (44) with (45) and (46) provides an estimate of the distribution of the registration parameters, where the mean (45) is utilized as their point estimate. An interesting observation is that this registration method is a generalized stochastic version of the LucasKanade registration algorithm [33] as applied to the super resolution problem. The classical Lucas-Kanade method can be obtained as a special case of (45) by setting the matrix $\Psi_{k}$ equal to zero. This matrix incorporates the uncertainty of the image estimate $\mathbf{x}$ into the motion estimation procedure. As will be demonstrated experimentally, this incorporation significantly helps in the motion estimation process and results in more accurate estimates, especially when the observation noise is high. Note that modifications on the classical Lucas-Kanade method (see, e.g., [39]) can also be incorporated into the proposed framework by appropriately manipulating the covariace matrix $\Psi_{k}$.

In this work, we considered a motion model that includes translation and rotation. However, the proposed framework is flexible enough to be extended to more complex parametric motion models, such as affine (with 6 degrees of freedom) or projective (with 8 degrees of freedom) motions. In these cases, we redefine the coordinate transformations in (4)-(5) appropriately and obtain $2 P N \times 6$ and $2 P N \times 8$ matrices for $\mathbf{P}\left(\overline{\mathbf{s}}_{k}\right)$ in (26), respectively. The rest of the corresponding motion estimation equations can then be derived with some algebra (explicit solutions for these motion models are not shown here for brevity).

$$
\Psi_{k}=\left(\begin{array}{ccc}
\operatorname{trace}\left[\mathbf{O}_{k 1}\left(\overline{\mathbf{s}}_{k}\right)^{T} \mathbf{O}_{k 1}\left(\overline{\mathbf{s}}_{k}\right) \Sigma_{\mathbf{x}}\right] & \operatorname{trace}\left[\mathbf{O}_{k 1}\left(\overline{\mathbf{s}}_{k}\right)^{T} \mathbf{O}_{k 2}\left(\overline{\mathbf{s}}_{k}\right) \Sigma_{\mathbf{x}}\right] & \operatorname{trace}\left[\mathbf{O}_{k 1}\left(\overline{\mathbf{s}}_{k}\right)^{T} \mathbf{O}_{k 3}\left(\overline{\mathbf{s}}_{k}\right) \Sigma_{\mathbf{x}}\right] \\
\operatorname{trace}\left[\mathbf{O}_{k 2}\left(\overline{\mathbf{s}}_{k}\right)^{T} \mathbf{O}_{k 1}\left(\overline{\mathbf{s}}_{k}\right) \Sigma_{\mathbf{x}}\right] & \operatorname{trace}\left[\mathbf{O}_{k 2}\left(\overline{\mathbf{s}}_{k}\right)^{T} \mathbf{O}_{k 2}\left(\overline{\mathbf{s}}_{k}\right) \Sigma_{\mathbf{x}}\right] & \operatorname{trace}\left[\mathbf{O}_{k 2}\left(\overline{\mathbf{s}}_{k}\right)^{T} \mathbf{O}_{k 3}\left(\overline{\mathbf{s}}_{k}\right) \Sigma_{\mathbf{x}}\right] \\
\operatorname{trace}\left[\mathbf{O}_{k 3}\left(\overline{\mathbf{s}}_{k}\right)^{T} \mathbf{O}_{k 1}\left(\overline{\mathbf{s}}_{k}\right) \Sigma_{\mathbf{x}}\right] & \operatorname{trace}\left[\mathbf{O}_{k 3}\left(\overline{\mathbf{s}}_{k}\right)^{T} \mathbf{O}_{k 2}\left(\overline{\mathbf{s}}_{k}\right) \Sigma_{\mathbf{x}}\right] & \operatorname{trace}\left[\mathbf{O}_{k 3}\left(\overline{\mathbf{s}}_{k}\right)^{T} \mathbf{O}_{k 3}\left(\overline{\mathbf{s}}_{k}\right) \Sigma_{\mathbf{x}}\right]
\end{array}\right)
$$

$$
\Phi_{k}=\left(\begin{array}{lll}
\mu_{\mathbf{x}}^{T} \mathbf{O}_{k 1}\left(\overline{\mathbf{s}}_{k}\right)^{T} \mathbf{O}_{k 1}\left(\overline{\mathbf{s}}_{k}\right) \mu_{\mathbf{x}} & \mu_{\mathbf{x}}^{T} \mathbf{O}_{k 1}\left(\overline{\mathbf{s}}_{k}\right)^{T} \mathbf{O}_{k 2}\left(\overline{\mathbf{s}}_{k}\right) \mu_{\mathbf{x}}^{T} & \mu_{\mathbf{x}}^{T} \mathbf{O}_{k 1}\left(\overline{\mathbf{s}}_{k}\right)^{T} \mathbf{O}_{k 3}\left(\overline{\mathbf{s}}_{k}\right) \mu_{\mathbf{x}} \\
\mu_{\mathbf{x}}^{T} \mathbf{O}_{k 2}\left(\overline{\mathbf{s}}_{k}\right)^{T} \mathbf{O}_{k 1}\left(\overline{\mathbf{s}}_{k}\right) \mu_{\mathbf{x}} & \mu_{\mathbf{x}}^{T} \mathbf{O}_{k 2}\left(\overline{\mathbf{s}}_{k}\right)^{T} \mathbf{O}_{k 2}\left(\overline{\mathbf{s}}_{k}\right) \mu_{\mathbf{x}} & \mu_{\mathbf{x}}^{T} \mathbf{O}_{k 2}\left(\overline{\mathbf{s}}_{k}\right)^{T} \mathbf{O}_{k 3}\left(\overline{\mathbf{s}}_{k}\right) \mu_{\mathbf{x}} \\
\mu_{\mathbf{x}}^{T} \mathbf{O}_{k 3}\left(\overline{\mathbf{s}}_{k}\right)^{T} \mathbf{O}_{k 1}\left(\overline{\mathbf{s}}_{k}\right) \mu_{\mathbf{x}} & \mu_{\mathbf{x}}^{T} \mathbf{O}_{k 3}\left(\overline{\mathbf{s}}_{k}\right)^{T} \mathbf{O}_{k 2}\left(\overline{\mathbf{s}}_{k}\right) \mu_{\mathbf{x}} & \mu_{\mathbf{x}}^{T} \mathbf{O}_{k 3}\left(\overline{\mathbf{s}}_{k}\right)^{T} \mathbf{O}_{k 3}\left(\overline{\mathbf{s}}_{k}\right) \mu_{\mathbf{x}}
\end{array}\right)
$$




\section{Estimation of the Hyperparameter Distributions}

In the last step of the algorithm, the distributions of the hyperparameters $\mathrm{q}\left(\alpha_{\mathrm{im}}\right)$ and $\mathrm{q}\left(\beta_{k}\right)$ are found from (22) as Gamma distributions, expressed as

$$
\begin{aligned}
& \mathrm{q}\left(\alpha_{\mathrm{im}}\right) \propto \alpha_{\mathrm{im}}^{P N / 2-1+a_{\alpha_{\mathrm{im}}}^{o}} \\
& \times \exp \left[-\alpha_{\mathrm{im}}\left(b_{\alpha_{\mathrm{im}}}^{o}+\sum_{i} \sqrt{w_{i}}\right)\right]
\end{aligned}
$$

and

$$
\begin{aligned}
& \mathrm{q}\left(\beta_{k}\right) \propto \beta_{k}^{N / 2-1+a_{\beta}^{o}} \\
& \quad \times \exp \left[-\beta_{k}\left(b_{\beta}^{o}+\frac{\mathrm{E}_{\mathbf{x}, \mathbf{s}_{k}}\left[\left\|\mathbf{y}_{k}-\mathbf{B}\left(\mathbf{s}_{k}\right) \mathbf{x}\right\|^{2}\right]}{2}\right)\right] .
\end{aligned}
$$

The quantity $\mathrm{E}_{\mathbf{x}, \mathbf{s}_{k}}\left[\left\|\mathbf{y}_{k}-\mathbf{B}\left(\mathbf{s}_{k}\right) \mathbf{x}\right\|^{2}\right]$ can be calculated using (35) as

$$
\begin{aligned}
\mathrm{E}_{\mathbf{x}, \mathbf{s}_{k}} & {\left[\left\|\mathbf{y}_{k}-\mathbf{B}\left(\mathbf{s}_{k}\right) \mathbf{x}\right\|^{2}\right] } \\
= & \mathrm{E}_{\mathbf{x}}\left[\mathrm{E}_{\mathbf{s}_{k}}\left[\left\|\mathbf{y}_{k}-\mathbf{B}\left(\mathbf{s}_{k}\right) \mathbf{x}\right\|^{2}\right]\right] \\
= & \mathrm{E}_{\mathbf{x}}\left[\left\|\mathbf{y}_{k}-\mathbf{B}\left(\overline{\mathbf{s}}_{k}\right) \mathbf{x}\right\|^{2}\right. \\
+ & \left.\sum_{i=1}^{3} \sum_{j=1}^{3} \lambda_{k i j} \mathbf{x}^{T} \mathbf{O}_{k i}\left(\overline{\mathbf{s}}_{k}\right)^{T} \mathbf{O}_{k j}\left(\overline{\mathbf{s}}_{k}\right) \mathbf{x}\right] \\
= & \left\|\mathbf{y}_{k}-\mathbf{B}\left(\overline{\mathbf{s}}_{k}\right) \mu_{\mathbf{x}}\right\|^{2}+\operatorname{trace}\left[\mathbf{B}\left(\overline{\mathbf{s}}_{k}\right)^{T} \mathbf{B}\left(\overline{\mathbf{s}}_{k}\right) \Sigma_{\mathbf{x}}\right] \\
& +\sum_{i=1}^{3} \sum_{j=1}^{3} \lambda_{k i j} \mu_{\mathbf{x}}^{T} \mathbf{O}_{k i}\left(\overline{\mathbf{s}}_{k}\right)^{T} \mathbf{O}_{k j}\left(\overline{\mathbf{s}}_{k}\right) \mu_{\mathbf{x}} \\
& +\operatorname{trace}\left[\sum_{i=1}^{3} \sum_{j=1}^{3} \lambda_{k i j} \mathbf{O}_{k i}\left(\overline{\mathbf{s}}_{k}\right)^{T} \mathbf{O}_{k j}\left(\overline{\mathbf{s}}_{k}\right) \Sigma_{\mathbf{x}}\right]
\end{aligned}
$$

The means of the distributions in (48) and (49), which are used as hyperparameter estimates, are given by

$$
\begin{aligned}
\left\langle\alpha_{\mathrm{im}}\right\rangle & =\frac{P N / 2+a_{\alpha_{\mathrm{im}}}^{o}}{\sum_{i} \sqrt{w_{i}}+b_{\alpha_{\mathrm{im}}}^{o}}, \\
\left\langle\beta_{k}\right\rangle & =\frac{N+2 a_{\beta}^{o}}{\mathrm{E}_{\mathbf{s}_{k}, \mathbf{x}}\left[\left\|\mathbf{y}_{k}-\mathbf{B}\left(\mathbf{s}_{k}\right) \mathbf{x}\right\|^{2}\right]+2 b_{\beta}^{o}} .
\end{aligned}
$$

Note that the shape and scale parameters $a_{\alpha_{\mathrm{im}}}^{o}, a_{\beta}^{o}, b_{\alpha_{\mathrm{im}}}^{o}, b_{\beta}^{o}$ can be used to incorporate prior knowledge about the variances of the HR image and observation noise, in case such knowledge is available. If they are set equal to $a_{\alpha_{\mathrm{im}}}^{o}=a_{\beta}^{o}=1$ and $b_{\alpha_{\text {im }}}^{o}=b_{\beta}^{o}=0$, which corresponds to utilizing flat hyperprior distributions for the hyperparameters, the observed LR images are made solely responsible for the whole estimation process.

In summary, the algorithm iterates between estimating the HR image using (37) and (38), the spatial adaptivity vector $\mathbf{w}$ using (40), the registration parameters using (45) and (46), and finally the hyperparameters using (51) and (52). The algorithm is summarized below in Algorithm 1. A major computational difficulty in Algorithm 1 is the explicit construction of the matrix $\Sigma_{\mathbf{x}}$ in (38), which requires the inversion of an $P N \times P N$ matrix. To avoid this computation, we solve (37) efficiently using the conjugate gradient method, and in equations where the explicit form of $\Sigma_{\mathbf{x}}$ is needed, i.e., in (40), (42), (43) and (50), $\Sigma_{\mathbf{x}}$ is approximated by a diagonal matrix obtained by inverting the diagonal elements of (38). We have conducted extensive experiments with small images which permit the explicit inversion of (38) to verify the validity of this approximation, and we found out empirically that this approximation results in very close estimates and has a minor effect in the estimation process. Similar approximations have also been utilized in other Bayesian recovery methods [15], [16], [30].

\section{Algorithm 1 Variational Bayesian Super Resolution}

Calculate initial estimates of the HR image, registration parameters, and hyperparameters

while convergence criterion is not met do

1. Compute the HR image distribution using (37) and (38).

2. Compute spatial adaptivity vector $\mathbf{w}$ using (40).

3. Compute the distribution of the registration parameters using (45) and (46).

4. Compute the distributions of the hyperparameters $\alpha_{\mathrm{im}}$, $\left\{\beta_{k}\right\}$ using (48) and (49).

It is worth emphasizing here that although we utilized the approximations (21) and (33) in computing their closed forms, we did not assume a priori that $\mathrm{q}(\mathbf{x})$ and $\mathrm{q}\left(\mathbf{s}_{k}\right)$ are Gaussian distributions. This result is derived due to the minimization of the KL divergence with respect to all possible distributions according to the factorization $\mathrm{q}\left(\alpha_{\mathrm{im}}\right) \mathrm{q}(\mathbf{x}) \prod_{k=1}^{L} \mathrm{q}\left(\mathbf{s}_{k}\right) \prod_{k=1}^{L} \mathrm{q}\left(\beta_{k}\right)$ [40]. We can, however, make an assumption that these distributions are degenerate, i.e., they take one value with probability one and the rest of the values with probability zero. Using this assumption, we obtain another algorithm very similar to the one presented above, with the only exception that the uncertainty terms arising from the covariance matrices are removed. The derivation of this algorithm is very similar to the first one, and therefore we omit its details and provide the iterative procedure below in Algorithm 2.

It is clear that using degenerate distributions for $\mathbf{x}$ and $\mathbf{s}_{k}$ in Algorithm 2 removes the uncertainty terms of the image and motion estimates. As demonstrated in the experimental results section, incorporation of this uncertainty through the covariances of $\mathbf{x}$ and $\mathbf{s}_{k}$ improves the restoration performance, especially in cases when the observation noise is high. This is mainly due to the fact that poor estimations of one variable (due to noise or outliers) can influence the estimation of other unknowns, and as a result the overall performance can significantly be affected. By estimating the full posterior distribution of the unknowns instead of point estimates corresponding to the maximum probability (such as MAP estimates), the uncertainty of the estimates 
is incorporated into the estimation procedure to ameliorate the propagation of estimation errors among unknowns.

\section{Algorithm 2 Variational Bayesian Super Resolution with Degenerate Distributions}

Calculate initial estimates of the initial HR image, registration parameters, and hyperparameters

while convergence criterion is not met do

1. Calculate the HR image estimate $\hat{\mathbf{x}}$ using

$$
\begin{aligned}
\hat{\mathbf{x}}= & {\left[\sum_{k} \hat{\beta}_{k} \mathbf{B}_{k}\left(\overline{\mathbf{s}}_{k}\right)^{T} \mathbf{B}_{k}\left(\overline{\mathbf{s}}_{k}\right)\right.} \\
& \left.+\hat{\alpha}_{\mathrm{im}}\left(\Delta^{h}\right)^{T} \mathbf{W} \Delta^{h}+\hat{\alpha}_{\mathrm{im}}\left(\Delta^{v}\right)^{T} \mathbf{W} \Delta^{v}\right]^{-1} \\
& \times\left[\sum_{k} \hat{\beta}_{k} \mathbf{B}_{k}\left(\overline{\mathbf{s}}_{k}\right)^{T} \mathbf{y}_{k}\right] .
\end{aligned}
$$

2. Compute spatial adaptivity vector $\mathbf{w}$ using

$$
w_{i}=\left(\Delta_{i}^{h}(\hat{\mathbf{x}})\right)^{2}+\left(\Delta_{i}^{v}(\hat{\mathbf{x}})\right)^{2} .
$$

3. Estimate registration parameters by solving

$$
\begin{array}{r}
\left(\overline{\mathbf{s}}_{k}\right)^{\text {new }}=\left[\left(\boldsymbol{\Lambda}_{k}^{p}\right)^{-1}+\hat{\beta}_{k} \Phi_{k}\right]^{-1}\left[\left(\boldsymbol{\Lambda}_{k}^{p}\right)^{-1} \overline{\mathbf{s}}_{k}^{p}+\hat{\beta}_{k} \Phi_{k} \overline{\mathbf{s}}_{k}\right. \\
\left.+\hat{\beta}_{k}\left(\begin{array}{c}
\left(\mathbf{y}_{k}-\mathbf{B}\left(\overline{\mathbf{s}}_{k}\right) \hat{\mathbf{x}}\right)^{T} \mathbf{O}_{k 1}\left(\overline{\mathbf{s}}_{k}\right) \hat{\mathbf{x}} \\
\left(\mathbf{y}_{k}-\mathbf{B}\left(\overline{\mathbf{s}}_{k}\right) \hat{\mathbf{x}}\right)^{T} \mathbf{O}_{k 2}\left(\overline{\mathbf{s}}_{k}\right) \hat{\mathbf{x}} \\
\left(\mathbf{y}_{k}-\mathbf{B}\left(\overline{\mathbf{s}}_{k}\right) \hat{\mathbf{x}}\right)^{T} \mathbf{O}_{k 3}\left(\overline{\mathbf{s}}_{k}\right) \hat{\mathbf{x}}
\end{array}\right)\right] .
\end{array}
$$

with (56), shown at the bottom of the page.

4. Compute hyperparameter estimates $\hat{\alpha}_{\text {im }}, \hat{\beta}_{k}$ using

$$
\begin{aligned}
\hat{\alpha}_{\mathrm{im}} & =\frac{P N / 2+a_{\alpha_{\mathrm{im}}}^{o}}{\sum_{i} \sqrt{w_{i}}+b_{\alpha_{\mathrm{im}}}^{o}}, \\
\hat{\beta}_{k} & =\frac{N+2 a_{\beta}^{o}}{\left\|\mathbf{y}_{k}-\mathbf{B}\left(\overline{\mathbf{s}}_{k}\right) \hat{\mathbf{x}}\right\|^{2}+2 b_{\beta}^{o}} .
\end{aligned}
$$

We conclude this section by commenting on the computational complexity of the algorithms. Algorithms 1 and 2 have similar complexities, with Algorithm 1 requiring more computations per iteration due to the incorporation of the covariance matrices. The majority of computations in both algorithms is performed for estimating the HR image and the registration vectors. The HR image is calculated efficiently using the conjugate gradient method [41] in (37) and (53), and the registration parameters are calculated by inverting a $3 \times 3$ matrix for each observed LR image in (45) and (55). Note that the matrix multiplications can be performed very efficiently by implementing the corresponding operators rather than storing full matrices. Therefore, the algorithms have computational demands very similar to most existing SR algorithms in the literature (for instance, the AM methods [10], [11], [16], [17]).

\section{EXPERIMENTAL RESULTS}

In this section, we analyze the performance of the proposed algorithms on both synthetic and real images under various conditions. In synthetic experiments, the quality of the restored HR image is measured quantitatively by the peak signal-to-noise ratio (PSNR), which is defined as

$$
\operatorname{PSNR}=10 \log _{10} \frac{N P}{\|\hat{\mathbf{x}}-\mathbf{x}\|^{2}}
$$

where $\hat{\mathbf{x}}$ and $\mathbf{x}$ are the estimated and original HR images, respectively, and pixel values in both images are normalized to lie in the interval $[0,1]$. We also provide examples of estimated HR images to assess their visual quality. To evaluate the estimated motion parameters we use the sum of squared errors (SSE), given by

$$
\mathrm{SSE}=\sum_{k=1}^{L}\left\|\hat{\mathbf{s}}_{k}-\mathbf{s}_{k}\right\|^{2}
$$

In the following, Algorithm 1 will be abbreviated as ALG1, and Algorithm 2 as $A L G 2$. In all experiments reported below, the initial values of the algorithms $A L G 1$ and $A L G 2$ are chosen as follows: The initial registration parameters are estimated using the standard Lucas-Kanade method [33] (similar results were obtained with other registration algorithms such as [32]). The HR image estimate is then initialized using the average image [17], which is an oversmooth estimate of the HR image obtained using the LR images as

$$
\mathbf{x}_{a}=\mathbf{S}^{-1} \sum_{k=1}^{L} \mathbf{B}_{k}\left(\overline{\mathbf{s}}_{k}\right)^{T} \mathbf{y}_{k}
$$

where $\mathbf{S}$ is a diagonal matrix with the column sums of $\mathbf{B}_{k}\left(\overline{\mathbf{s}}_{k}\right)$ as its elements. Note that this initial estimate is calculated very efficiently, and it generally increases the robustness of the algorithm to the noise. On the other hand, other initializations (such as bicubic interpolation) resulted in similar restorations.

The inverse covariance matrices $\left(\boldsymbol{\Lambda}_{k}^{p}\right)^{-1}$ are set equal to zero matrices, that is, no prior information is utilized about the uncertainty of motion vectors. The covariance matrices in $A L G 1$ are initially set equal to zero. The rest of the algorithm parameters are automatically calculated from the initial HR image estimate using the algorithm steps provided in Algorithm 1 and Algorithm 2. As the convergence criterion we used $\left\|\mathrm{x}^{n}-\mathrm{x}^{n-1}\right\|^{2} /\left\|\mathrm{x}^{n-1}\right\|^{2}<10^{-5}$, where $\mathrm{x}^{n}$ and $\mathrm{x}^{n-1}$ are the image estimates at the $n$th and $(n-1)$ st iterations, respectively.

$$
\Phi_{k}=\left(\begin{array}{ccc}
\hat{\mathbf{x}}^{T} \mathbf{O}_{k 1}\left(\overline{\mathbf{s}}_{k}\right)^{T} \mathbf{O}_{k 1}\left(\overline{\mathbf{s}}_{k}\right) \hat{\mathbf{x}} & \hat{\mathbf{x}}^{T} \mathbf{O}_{k 1}\left(\overline{\mathbf{s}}_{k}\right)^{T} \mathbf{O}_{k 2}\left(\overline{\mathbf{s}}_{k}\right) \hat{\mathbf{x}}^{T} & \hat{\mathbf{x}}^{T} \mathbf{O}_{k 1}\left(\overline{\mathbf{s}}_{k}\right)^{T} \mathbf{O}_{k 3}\left(\overline{\mathbf{s}}_{k}\right) \hat{\mathbf{x}} \\
\hat{\mathbf{x}}^{T} \mathbf{O}_{k 2}\left(\overline{\mathbf{s}}_{k}\right)^{T} \mathbf{O}_{k 1}\left(\overline{\mathbf{s}}_{k}\right) \hat{\mathbf{x}} & \hat{\mathbf{x}}^{T} \mathbf{O}_{k 2}\left(\overline{\mathbf{s}}_{k}\right)^{T} \mathbf{O}_{k 2}\left(\overline{\mathbf{s}}_{k}\right) \hat{\mathbf{x}} & \hat{\mathbf{x}}^{T} \mathbf{O}_{k 2}\left(\overline{\mathbf{s}}_{k}\right)^{T} \mathbf{O}_{k 3}\left(\overline{\mathbf{s}}_{k}\right) \hat{\mathbf{x}} \\
\hat{\mathbf{x}}^{T} \mathbf{O}_{k 3}\left(\overline{\mathbf{s}}_{k}\right)^{T} \mathbf{O}_{k 1}\left(\overline{\mathbf{s}}_{k}\right) \hat{\mathbf{x}} & \hat{\mathbf{x}}^{T} \mathbf{O}_{k 3}\left(\overline{\mathbf{s}}_{k}\right)^{T} \mathbf{O}_{k 2}\left(\overline{\mathbf{s}}_{k}\right) \hat{\mathbf{x}} & \hat{\mathbf{x}}^{T} \mathbf{O}_{k 3}\left(\overline{\mathbf{s}}_{k}\right)^{T} \mathbf{O}_{k 3}\left(\overline{\mathbf{s}}_{k}\right) \hat{\mathbf{x}}
\end{array}\right) .
$$




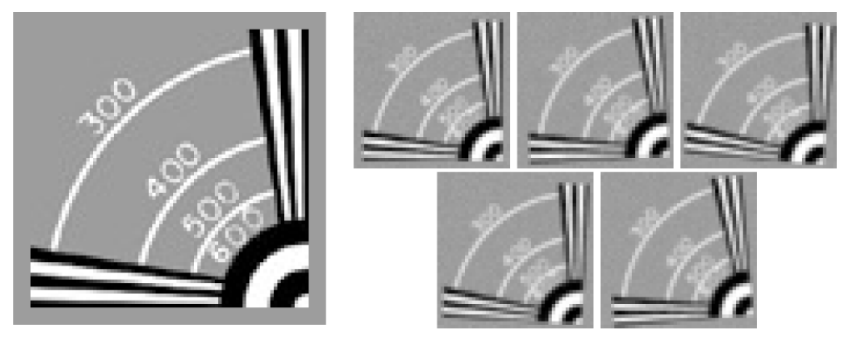

Fig. 1. (Left) Original HR image. (Right) Five synthetically generated LR images.

In the following subsections, we present experimental results demonstrating the performance limits of the proposed algorithms with known motion information, robustness to initial registration inaccuracy, the effect of the number of LR images on the quality of the estimated HR image, and reconstruction performance with real images, in comparison with existing approaches.

\section{A. Synthetic Experiments With Exact Motion Information}

In this section, we evaluate the performance of two SR methods in comparison with the proposed algorithms in cases where exact motion information is available. This study presents a comparison of the best possible performances achieved by the algorithms, and in addition it provides reference information to evaluate their behavior when the motion information is inaccurate, which will be studied in the next subsection.

We used the following methods for comparison: 1) Bicubic interpolation, 2) the robust SR method in [5] (denoted by ZMT), which is based on backprojection with median filtering, and 3) the robust SR method in [6] (denoted by RSR), which is based on bilateral TV priors. We also experimented with other SR methods contained in the EPFL SR software [42], but they provided inferior results compared to $Z M T$ and $R S R$, and therefore they are not reported here.

We generated 5 synthetic LR images from the HR image shown on the left in Fig. 1 through warping, blurring and downsampling by a factor of 2 . The warping consists of both translation and rotation, where the translations are chosen as

$$
\left(\begin{array}{c}
0.0 \\
0.0
\end{array}\right),\left(\begin{array}{c}
0.0 \\
0.5
\end{array}\right),\left(\begin{array}{l}
0.5 \\
0.0
\end{array}\right),\left(\begin{array}{l}
1.0 \\
0.0
\end{array}\right),\left(\begin{array}{l}
0.0 \\
1.0
\end{array}\right)
$$

pixels, and the rotation angles are $\left(0^{\circ}, 3^{\circ},-3^{\circ}, 5^{\circ},-5^{\circ}\right)$, respectively. As the blur we used a $3 \times 3$ uniform PSF. The LR images obtained after the warping, blurring and downsampling operations are further degraded by additive white Gaussian noise at SNR levels of $5 \mathrm{~dB}, 15 \mathrm{~dB}, 25 \mathrm{~dB}, 35 \mathrm{~dB}$ and $45 \mathrm{~dB}$. Example LR images corresponding to the $25 \mathrm{~dB}$ SNR case are shown in Fig. 1. Note that this resolution chart image is chosen for better illustration of the performance in resolution enhancement; similar results were obtained in experiments with other images.

We conducted simulations with 20 different noise realizations at each SNR level, and the average and variance of these experiments are reported. Since the algorithms ZMT and $R S R$ contain algorithmic parameters, we exhaustively searched for the parameters resulting in the maximum PSNR value to report their best performance. Moreover, we reported the maximum PSNR

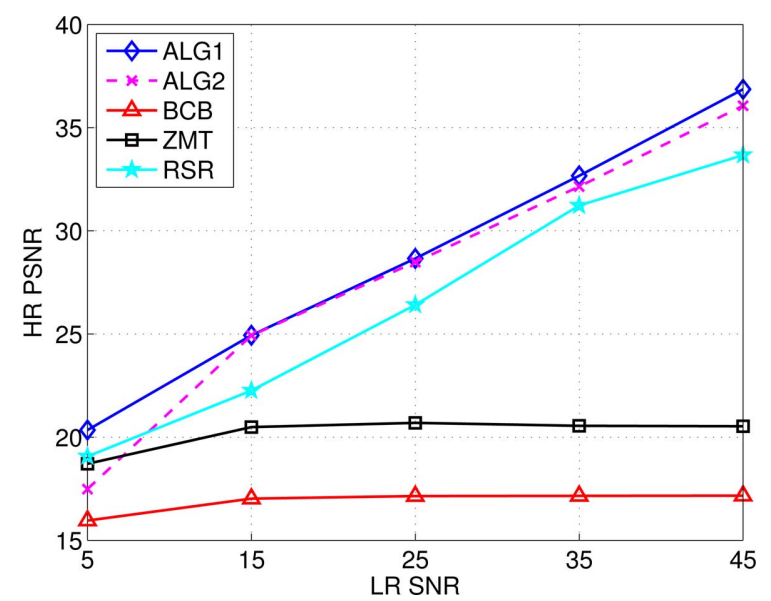

Fig. 2. Mean PSNR values of SR algorithms for different input SNR levels when exact motion information is available.

result obtained during their iterations rather than the PSNR result at convergence, and initialized the algorithms with both the bicubic interpolation result and the average image in (61), and chose the best resulting image among them. Note, however, that the parameters of the proposed methods are estimated automatically so there is no need for parameter tuning.

Mean PSNR values with the standard deviations provided by the algorithms are shown in Table I, and the mean PSNR values are plotted in Fig. 2. As expected, all SR algorithms result in better reconstructions than bicubic interpolation. It is also clear that the proposed methods provide the best performance among all methods across all noise levels. It should be emphasized that the PSNR values of the methods $Z M T$ and $R S R$ are obtained by exhaustively adjusting their parameters, which requires multiple runs, whereas the proposed methods provided their results in an fully-automated fashion in a single run. Therefore, even in the cases where the PSNR values are close, algorithms $A L G 1$ and $A L G 2$ should be preferred as the method of choice.

In general, $A L G 1$ provides restored HR images with slightly higher quality than $A L G 2$. This is especially evident in highnoise cases (e.g., SNR $=5 \mathrm{~dB}$ ), where the incorporation of the uncertainty prevents the algorithm from overfitting due to high noise.

Example HR restorations are shown in Fig. 3 for the SNR = $25 \mathrm{~dB}$ case, and in Fig. 4 for the SNR $=45 \mathrm{~dB}$ case. It is clear that the proposed methods provide the most visually enhanced restorations with significantly reduced ringing artifacts and much sharper edges compared to other methods. Restorations provided by $A L G 1$ and $A L G 2$ are very similar, with $A L G 1$ providing slightly sharper edges with less ringing artifacts.

\section{B. Synthetic Experiments With Inaccurate Motion Information}

In this section, we compare the performance of the SR methods when the motion parameters are inaccurate. We utilized the same setup as in the previous section, and used the same datasets to measure the decrease in performance due to the errors in registration parameters. In order to simulate the errors in motion estimation, we corrupted the original translation parameters by white Gaussian noise with standard 
TABLE I

Mean PSNRs With Standard DeViations in 20 Experiments PRovided by the SR Algorithms AT DIFFERENT SNR LEVELS WHEN MOTION INFORMATION IS EXACT

\begin{tabular}{|l|c|c|c|c|c|}
\hline SNR & $5 \mathrm{~dB}$ & $15 \mathrm{~dB}$ & $25 \mathrm{~dB}$ & $35 \mathrm{~dB}$ & $45 \mathrm{~dB}$ \\
\hline Bicubic & $15.96 \pm 0.077$ & $17.02 \pm 0.027$ & $17.14 \pm 0.008$ & $17.16 \pm 0.003$ & $17.16 \pm 0.001$ \\
\hline ZMT & $18.71 \pm 0.082$ & $20.48 \pm 0.242$ & $20.69 \pm 0.378$ & $20.55 \pm 0.275$ & $20.53 \pm 0.002$ \\
\hline RSR & $19.07 \pm 0.075$ & $22.25 \pm 0.049$ & $26.4 \pm 0.080$ & $31.22 \pm 0.059$ & $33.67 \pm 0.070$ \\
\hline ALG1 & $20.34 \pm 0.049$ & $24.93 \pm 0.130$ & $28.66 \pm 0.119$ & $32.67 \pm 0.110$ & $36.85 \pm 0.142$ \\
\hline ALG2 & $17.48 \pm 0.049$ & $24.93 \pm 0.123$ & $28.48 \pm 0.112$ & $32.15 \pm 0.092$ & $36.05 \pm 0.155$ \\
\hline
\end{tabular}

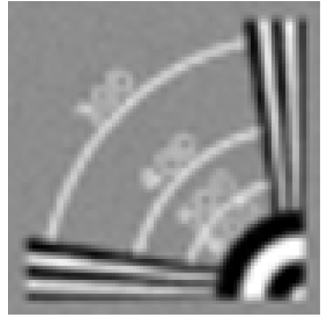

(a)

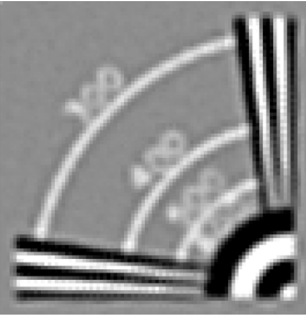

(b)

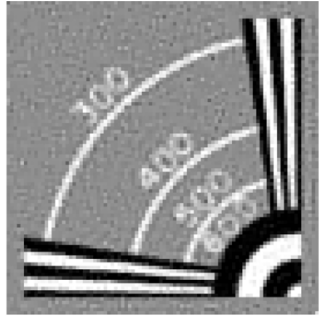

(c)

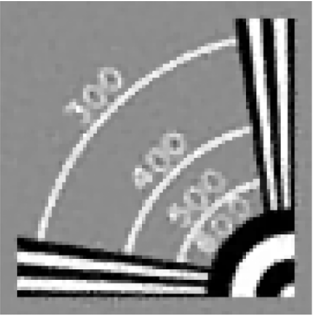

(d)

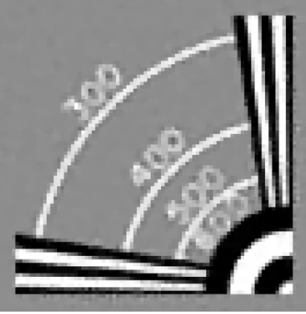

(e)

Fig. 3. Example estimated HR images from different SR methods in the case when SNR $=25 \mathrm{~dB}$ and motion information is exact. Results of (a) Bicubic interpolation $(\mathrm{PSNR}=17.14 \mathrm{~dB}),(\mathrm{b}) Z M T(\mathrm{PSNR}=20.55 \mathrm{~dB}),(\mathrm{c}) R S R(\mathrm{PSNR}=26.41 \mathrm{~dB})$, and the proposed methods $(\mathrm{d}) A L G 1(\mathrm{PSNR}=28.75 \mathrm{~dB})$, and $(\mathrm{e}) A L G 2(\mathrm{PSNR}=28.58 \mathrm{~dB})$.

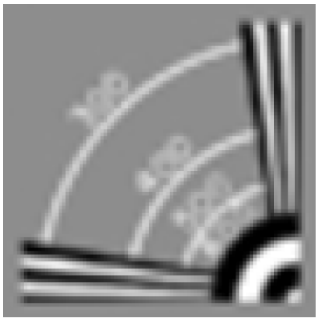

(a)

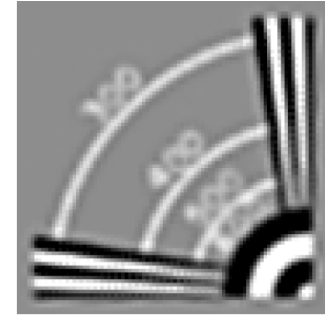

(b)

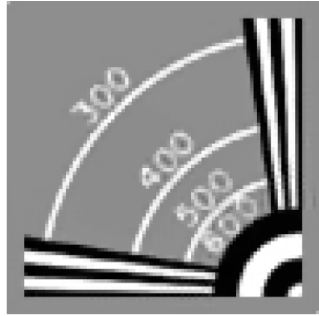

(c)

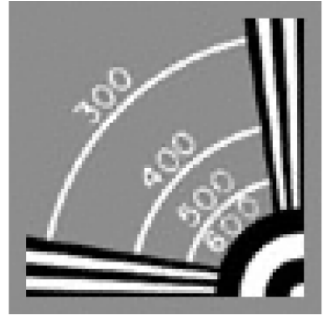

(d)

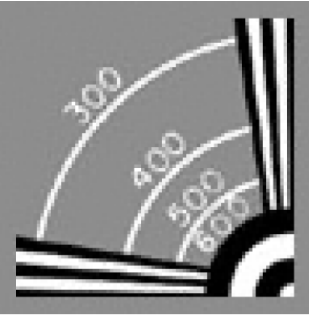

(e)

Fig. 4. Example estimated HR images from different SR methods in the case when SNR $=45 \mathrm{~dB}$ and motion information is exact. Results of (a) Bicubic interpolation $(\mathrm{PSNR}=17.16 \mathrm{~dB}),(\mathrm{b}) Z M T(\mathrm{PSNR}=20.53 \mathrm{~dB}),(\mathrm{c}) R S R(\mathrm{PSNR}=33.56 \mathrm{~dB})$, and the proposed methods $(\mathrm{d})$ ALG1 $(\mathrm{PSNR}=36.81 \mathrm{~dB})$, and (e) $A L G 2(\mathrm{PSNR}=35.85 \mathrm{~dB})$.

TABLE II

MEAN PSNRs With STANDARd DEVIATIONS IN 20 EXPERIMENTS PROVIDEd By THE SR Algorithms AT DiFFERENT SNR LEVELS WHEN MOTION INFORMATION IS INACCURATE (SEE TEXT)

\begin{tabular}{|l|c|c|c|c|c|}
\hline SNR & $5 \mathrm{~dB}$ & $15 \mathrm{~dB}$ & $25 \mathrm{~dB}$ & $35 \mathrm{~dB}$ & $45 \mathrm{~dB}$ \\
\hline Bicubic & $15.97 \pm 0.077$ & $17.02 \pm 0.027$ & $17.14 \pm 0.008$ & $17.16 \pm 0.003$ & $17.16 \pm 0.001$ \\
\hline ZMT & $16.26 \pm 0.978$ & $16.86 \pm 1.035$ & $17.76 \pm 0.503$ & $17.84 \pm 0.552$ & $17.88 \pm 0.600$ \\
\hline RSR & $16.84 \pm 0.412$ & $17.81 \pm 0.567$ & $18.02 \pm 0.655$ & $18.07 \pm 0.688$ & $18.08 \pm 0.692$ \\
\hline NLS & $17.16 \pm 0.071$ & $22.44 \pm 0.093$ & $25.37 \pm 0.132$ & $29.05 \pm 0.140$ & $32.39 \pm 0.183$ \\
\hline ALG1 & $19.83 \pm 0.050$ & $24.89 \pm 0.132$ & $28.44 \pm 0.154$ & $32.06 \pm 0.151$ & $35.58 \pm 0.330$ \\
\hline ALG2 & $17.49 \pm 0.050$ & $24.94 \pm 0.127$ & $28.34 \pm 0.115$ & $31.57 \pm 0.107$ & $33.86 \pm 0.416$ \\
\hline
\end{tabular}

deviation of 1 , and the rotation parameters with noise uniformly distributed in $\left[-2^{\circ}, 2^{\circ}\right]$. We also compare the proposed methods with the method proposed in [21], denoted by $N L S$, which simultaneously estimates both the HR image and the registration parameters. Note that this algorithm has two free parameters, which we have manually tuned for each noise setting and reported its best performance.

Mean PSNR values with standard deviations in 20 experiments are reported in Table II, and the mean PSNR values are plotted in Fig. 5. Comparing Tables I and II, it can be seen that 
TABLE III

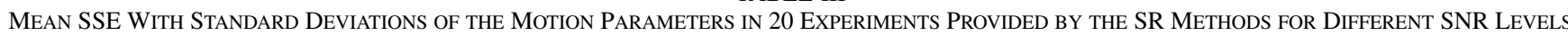

\begin{tabular}{|l|c|c|c|c|c|}
\hline & \multicolumn{5}{|c|}{ SNR } \\
\hline Methods & $5 \mathrm{~dB}$ & $15 \mathrm{~dB}$ & $25 \mathrm{~dB}$ & $35 \mathrm{~dB}$ & $45 \mathrm{~dB}$ \\
\hline NLS & $0.1659 \pm 0.0096$ & $0.0054 \pm 0.0029$ & $0.0006 \pm 0.0004$ & $0.0001 \pm 1 \times 10^{-4}$ & $7.29 \times 10^{-6} \pm 1.0 \times 10^{-5}$ \\
\hline ALG1 & $0.1436 \pm 0.0182$ & $0.0095 \pm 0.0026$ & $0.0027 \pm 0.0008$ & $0.0003 \pm 1 \times 10^{-4}$ & $3.17 \times 10^{-5} \pm 1.0 \times 10^{-5}$ \\
\hline ALG2 & $0.1451 \pm 0.0315$ & $0.0016 \pm 0.0006$ & $0.0003 \pm 0.0001$ & $0.0015 \pm 5 \times 10^{-5}$ & $2.60 \times 10^{-3} \pm 1.1 \times 10^{-3}$ \\
\hline
\end{tabular}

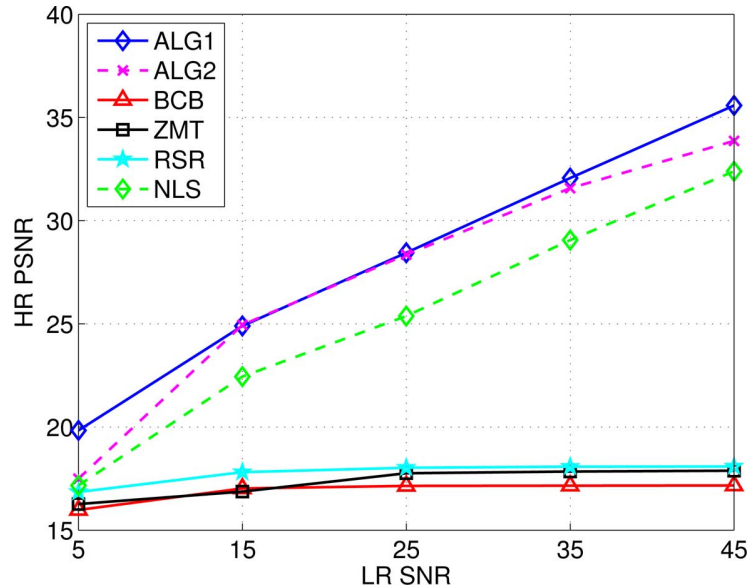

Fig. 5. Mean PSNR values of SR algorithms for different input SNR levels when motion information is inaccurate.

the performance of all algorithms decrease due to the motion errors, as expected. However, the performance degradation is severe with algorithms $Z M T$ and $R S R$, mainly due to the fact that they do not incorporate motion estimation, but try to compensate for the motion errors using robust observation models. On the other hand, it is clear from Table II and Fig. 5 that the performance degradation with algorithms $A L G 1$ and $A L G 2$ is minor, and they resulted in almost the same PSNR values as in the case when motion information is exact. This indicates that the restoration quality is significantly improved when the motion is accurately estimated. The $N L S$ method also outperforms the ZMT and RSR algorithms due to the joint estimation of motion parameters. However, it provides lower PSNR values than $A L G 1$ and $A L G 2$ in all noise levels although it requires user-supervision whereas the proposed methods are fully-automated.

The corresponding SSE values of the motion parameters estimated by $A L G 1, A L G 2$ and $N L S$ are shown in Table III. Note that $A L G 1$ and $A L G 2$ estimate the motion parameters very accurately in all noise levels. The $N L S$ method also results in very accurate motion estimates; some of its results are slightly better than the ones of the proposed methods, although all three algorithms resulted in negligibly small errors. Examples of estimated motion parameters are shown in Fig. 6 for the SNR $=25$ $\mathrm{dB}$ case, and in Fig. 7 for the SNR $=45 \mathrm{~dB}$ case. Note that in both cases the algorithms provide very accurate estimates of the motion vectors, even though the initial vectors contain high amounts of noise. Another observation is that the variances among the resulting PSNR values obtained by $A L G 1$ and $A L G 2$ (a)

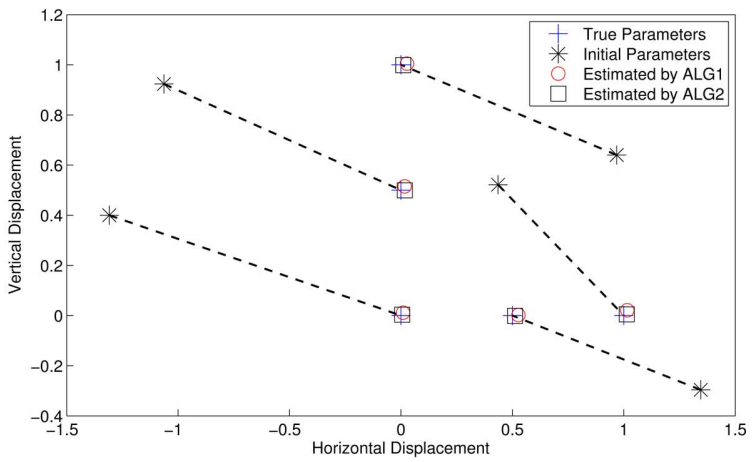

(b)

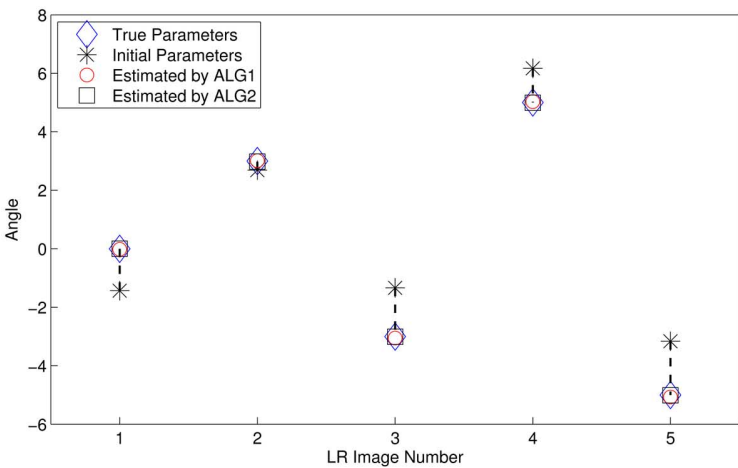

Fig. 6. Comparison between the true and initial motion parameters and motion parameters estimated by the algorithms $A L G 1$ and $A L G 2$ when SNR $=25 \mathrm{~dB}$. (a) Translation parameters. (b) Rotation angles. The resulting SSEs of the estimated parameters are $2.9 \times 10^{-3}$ for $A L G 1$ and $7.91 \times 10^{-4}$ for $A L G 2$. The initial and estimated motion parameter pairs for each LR image are connected by dashed lines.

are much smaller than the PSNR variances obtained by $Z M T$ and $R S R$, and very similar to the ones provided by $N L S$, indicating the robustness of the proposed methods to inaccurate initialization of motion parameters.

Examples of HR images estimated by the algorithms are shown in Fig. 8 for the SNR $=25 \mathrm{~dB}$ case, and in Fig. 9 for the SNR $=45 \mathrm{~dB}$ case. The degradation of visual quality in the methods $Z M T$ and $R S R$ is clear, especially comparing Figs. 3(b)-(c) to 8(b)-(c), and 4(b)-(c) to 9(b)-(c), respectively. On the other hand, $A L G 1$ and $A L G 2$ provided very high quality restorations, and there is almost no quality degradation when the initial motion parameters are inaccurate (compare Figs. 3(d)-(e) to 8(d)-(e), and 4(d)-(e) to 9(d)-(e), respectively, for $A L G 1$ and $A L G 2$ ). The $N L S$ method also provided results with much higher quality than ZMT and $R S R$, but they contain a higher level of ringing artifacts compared to the images provided by $A L G 1$ and $A L G 2$. 
(a)
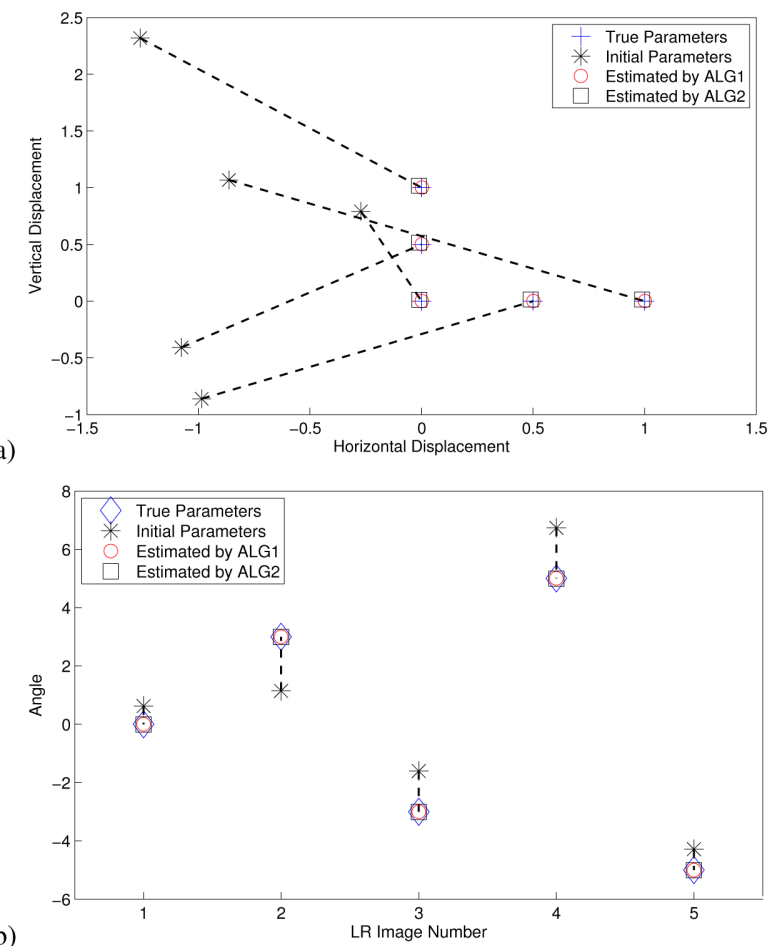

(b)

Fig. 7. Comparison between the true and initial motion parameters and motion parameters estimated by the algorithms $A L G 1$ and $A L G 2$ when SNR $=45 \mathrm{~dB}$. (a) Translation parameters. (b) Rotation angles. The resulting SSEs of the estimated parameters are $2.48 \times 10^{-5}$ for $A L G 1$ and $1.7 \times 10^{-3}$ for $A L G 2$. The initial and estimated motion parameter pairs for each LR image are connected by dashed lines.

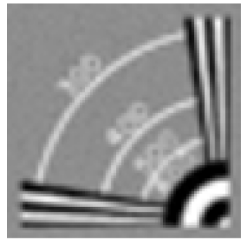

(a)

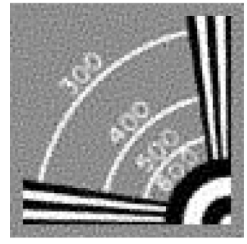

(d)

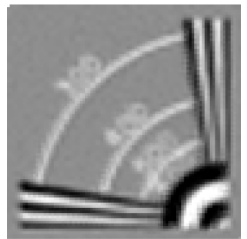

(b)

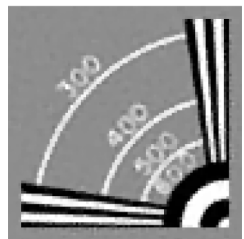

(e)

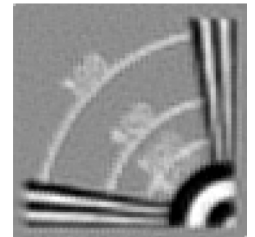

(c)

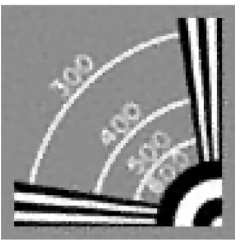

(f)
Fig. 8. Example estimated HR images from different SR methods in the case when SNR $=25 \mathrm{~dB}$ and motion information is inaccurate (see text). Results of (a) Bicubic interpolation $($ PSNR $=17.14 \mathrm{~dB}),($ b) $Z M T(P S N R=17.47 \mathrm{~dB})$, (c) $R S R(\mathrm{PSNR}=17.41 \mathrm{~dB}),(\mathrm{d}) N L S(\mathrm{PSNR}=25.62 \mathrm{~dB})$, and the proposed methods (e) $A L G 1(\mathrm{PSNR}=28.47 \mathrm{~dB})$, and $(\mathrm{f}) A L G 2(\mathrm{PSNR}=28.34 \mathrm{~dB})$.

\section{Effect of Number of LR Images}

In this section we present a study of the effect of the number of LR images on the quality of the estimated HR image. To provide results with realistic images, we generated LR images from the well-known "Cameraman" image by a downsampling factor of 4 and a $3 \times 3$ uniform blur kernel. The number of LR images are varied from 10 to 20 . The translation parameters are chosen randomly between 0 and 8 , and the rotation angles are

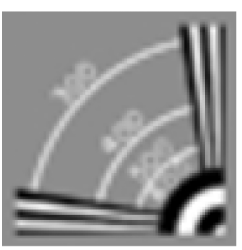

(a)

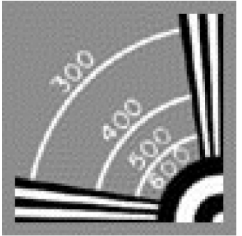

(d)

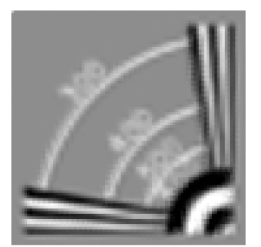

(b)

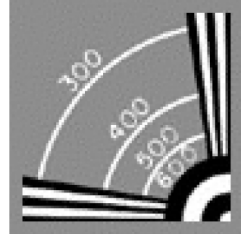

(e)

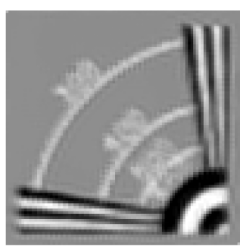

(c)

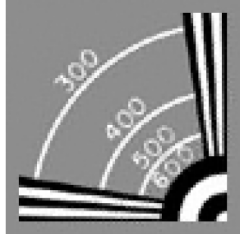

(f)
Fig. 9. Example estimated HR images from different SR methods in the case when $\mathrm{SNR}=45 \mathrm{~dB}$ and motion information is inaccurate (see text). Results of (a) Bicubic interpolation (PSNR $=17.16 \mathrm{~dB})$, (b) ZMT (PSNR $=17.49 \mathrm{~dB}$ ) (c) $R S R(\mathrm{PSNR}=17.44 \mathrm{~dB}),(\mathrm{d}) N L S(\mathrm{PSNR}=32.65 \mathrm{~dB})$, and the proposed methods (e) $A L G 1(\mathrm{PSNR}=35.11 \mathrm{~dB})$, and $(\mathrm{f}) A L G 2(\mathrm{PSNR}=33.40 \mathrm{~dB})$.

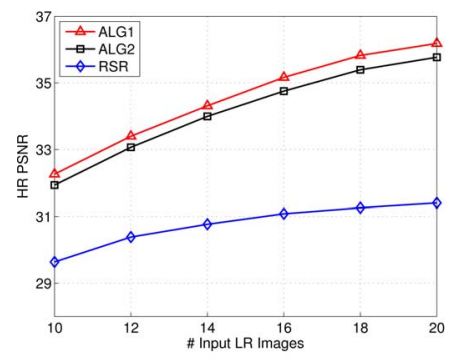

(a)

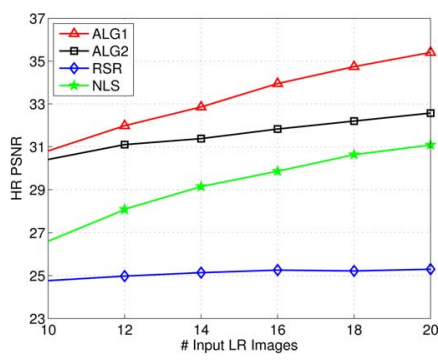

(b)
Fig. 10. Number of LR images vs PSNR of the estimated HR images for the "Cameraman" image with resolution increase of 4 (a) when exact motion information is available, and (b) when initial motion information is inaccurate.

chosen randomly in $\left[-5^{\circ}, 5^{\circ}\right]$. We experimented with both exact and inaccurate initialization of the motion parameters. The $N L S$ method is included only with the inaccurate motion case, since when exact motion information is available, it reduces to a special case of $A L G 2$ without parameter estimation.

Average PSNR results of 20 different motion and noise realizations for the exact motion information case are shown in Fig. 10(a). As expected, the performance of all algorithms increase as the number of images increase. The methods $A L G 1$ and $A L G 2$ clearly outperform $R S R$ independent of the number of LR images. Their performance is very close and they provide very high PSNR results even when the number of LR images is relatively low (e.g., 10).

As shown in the previous section, the performance of the proposed methods does not significantly reduce when the motion information is inaccurate. Average PSNR results for this case are shown in Fig. 10(b). A few important observations can be made: The performance of the RSR algorithm does not increase significantly with increasing number of LR images due to highly inaccurate motion parameters. Second, $A L G 1$ is more capable than $A L G 2$ with handling inaccurate initial motion parameters, and its performance is very close to the exact motion information case. Finally, although the $N L S$ algorithm also outperforms the RSR method, its performance is inferior to both 

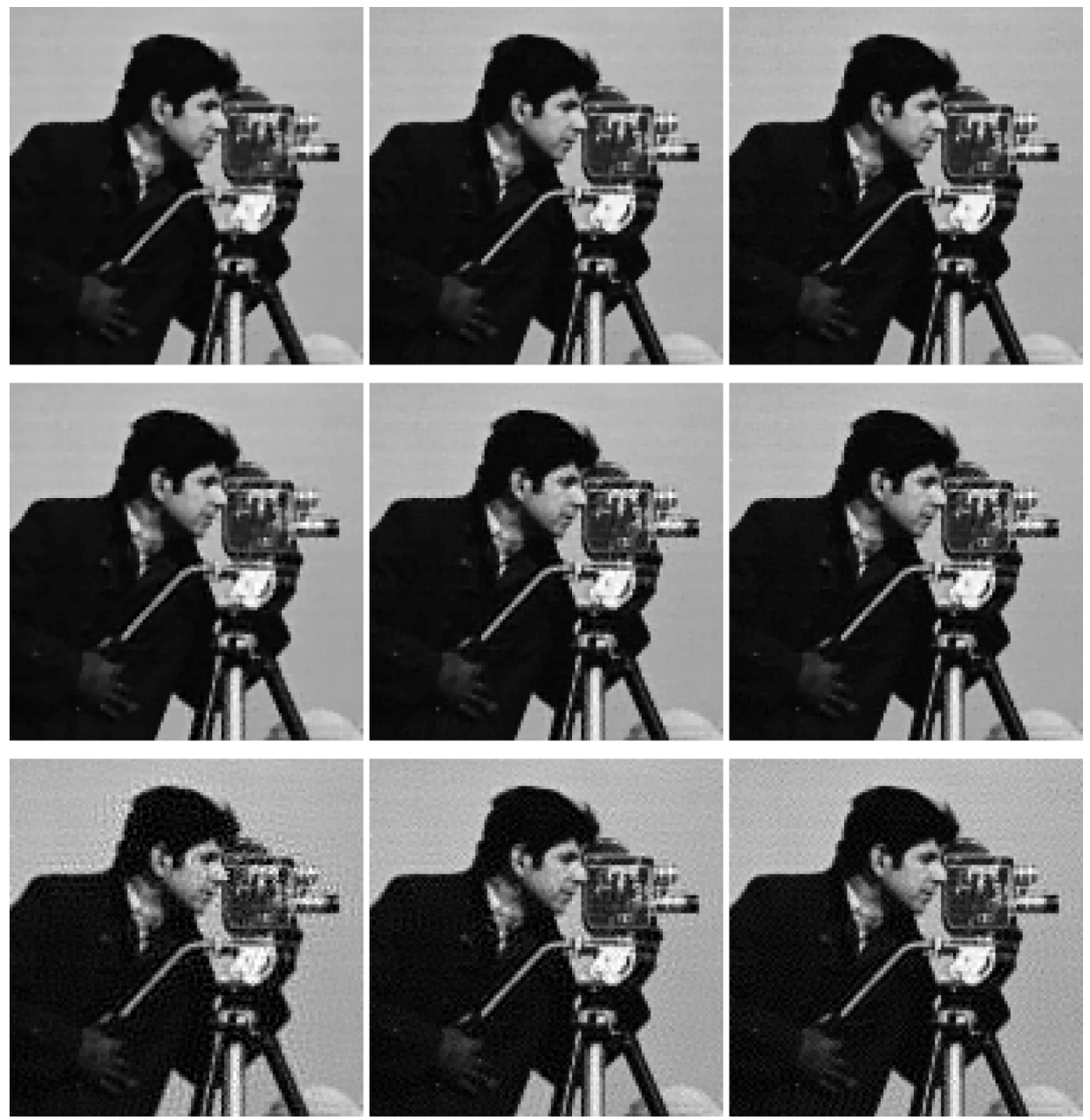

Fig. 11. Midsections of example reconstructed HR images with different number of input LR images when the initial motion information is inaccurate. Left column: with 10; middle column: with 16; and right column: with 20 LR images. Top row: results of algorithm $A L G 1$; middle row: results of algorithm $A L G 2$; and bottom row: results of algorithm $N L S$. The corresponding PSNR values are (in $\mathrm{dB}$ ) $31.10,34.26,35.60$ for $A L G 1,30.569,32.16,32.95$ for $A L G 2$, and 27.80, $30.72,31.37$ for $N L S$.

$A L G 1$ and $A L G 2$. For visual comparison, example reconstructed HR images are shown in Fig. (11) obtained by algorithms $A L G 1$, $A L G 2$, and $N L S$.

\section{Experiments With Real Images}

We conducted extensive experiments with the proposed algorithms on real SR applications, some of which are presented in this section. We report real image experiments performed on the datasets provided by UCSC [43]. The algorithms ZMT and $R S R$ are used again for comparing the performance of the algorithms, and we used the MDSP software [44] to obtain their results. We compare the proposed methods with $R S R, N L S$ and with the algorithm in [4], denoted by $E F$. The motion parameters are estimated from the LR images using the MDSP software, and provided to $N L S, A L G 1, A L G 2$ as initial parameters.

As with the synthetic experiments, we manually tuned all required parameters of the algorithms $R S R, N L S$ and $E F$ to obtain the most visually appealing results. On the other hand, no prior knowledge is assumed in the proposed methods except for the initial motion parameters. The inverse covariance matrices $\left(\Lambda_{k}^{p}\right)^{-1}$ are set equal to zero matrices so that the estimation process only depends on the LR images.

In the first experiment, 20 LR images were used taken from the disk dataset from [43]. The blur PSF is assumed to be a $5 \times 5$ Gaussian with variance 1 . The reconstructed HR images by a factor of four resolution enhancement obtained by bicubic interpolation and SR algorithms are shown in Fig. 12.

The second dataset consists of 15 LR images taken from the Adyoron dataset from [43]. The blur PSF is again assumed to be a $5 \times 5$ Gaussian with variance 1 . The reconstructed HR images by a factor of three resolution enhancement obtained by bicubic interpolation and SR algorithms are shown in Fig. 13.

It is clear from Figs. 12 and 13 that the proposed methods provide HR image estimates with sharper edges and fewer ringing artifacts than other methods. This is especially clear around the edges and around the letters in both images. Another observation is that the proposed methods are very effective in preserving sharp image features while suppressing noise and motion artifacts. $A L G 1$ and $A L G 2$ provide very similar results, but 


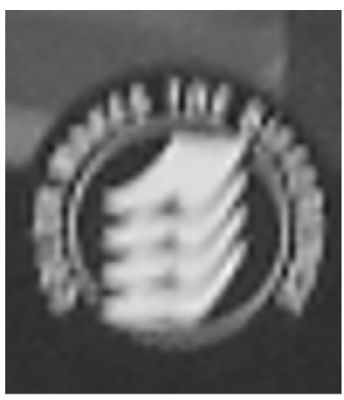

(a)

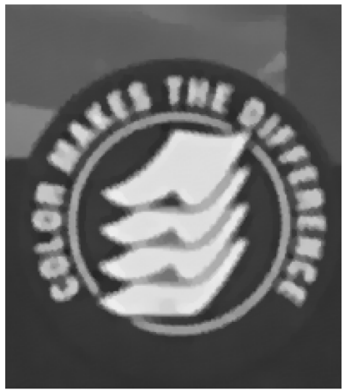

(c)

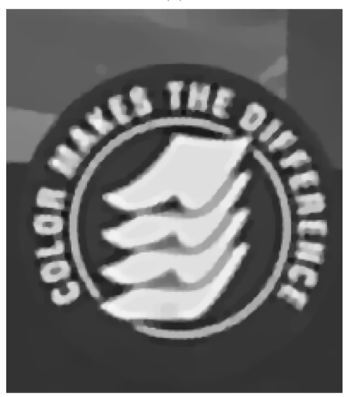

(e)

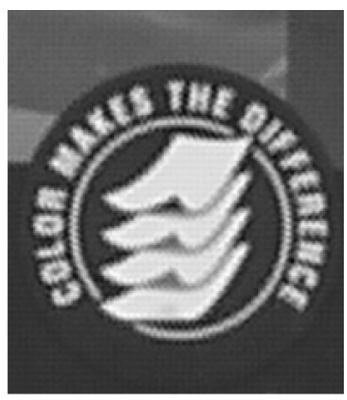

(b)

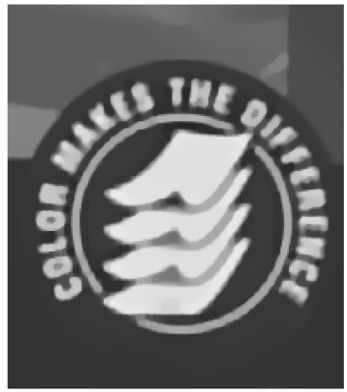

(d)

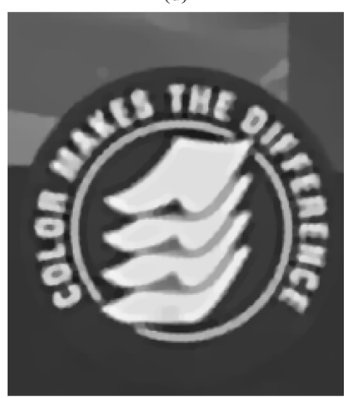

(f)
Fig. 12. Super resolution results (4x resolution increase) by (a) bicubic interpolation, (b) $E F$, (c) $R S R$, (d) $N L S$, (e) $A L G 1$ and (f) $A L G 2$.

ALG1 results in slightly sharper images and the ringing artifacts around the edges are more suppressed than in the results of $A L G 2$.

Finally, we show the application of the proposed methods to a real face data-set. The dataset contains 40 LR images of size $50 \times 50$. The reconstructed HR images by a factor of eight resolution increase obtained by bicubic interpolation and SR algorithms are shown in Fig. (14). Although the proposed algorithms are not specifically designed for face images, and the resolution enhancement factor is large, the reconstructed images are of high quality and visually better than those provided by other methods.

In summary, experimental results with both synthetic and real image sets demonstrate that the proposed algorithms are very effective in providing high quality super resolution results, and they compare favorably to some of the state-of-the-art super resolution methods.

\section{CONCLUSION}

In this paper, we presented a novel Bayesian formulation for joint image registration and super resolution. The unknown high resolution image, motion parameters and algorithm parameters,

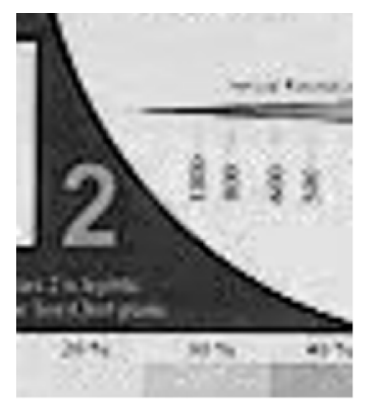

(a)

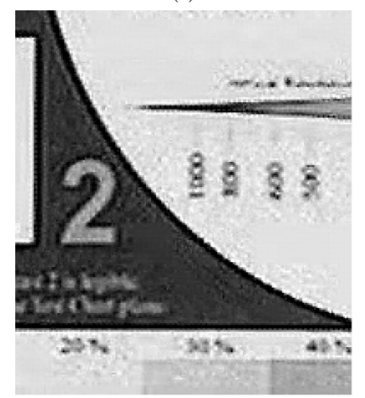

(c)

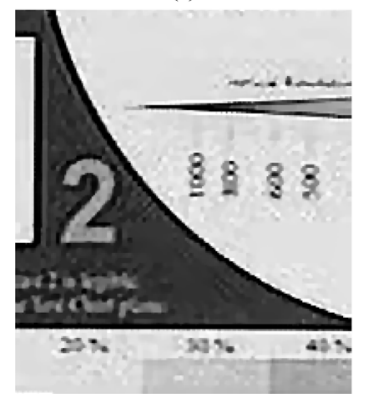

(e)

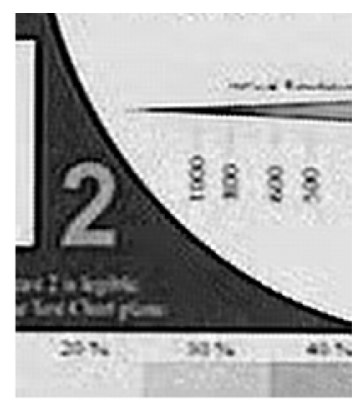

(b)

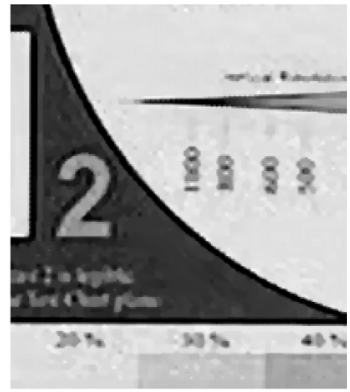

(d)

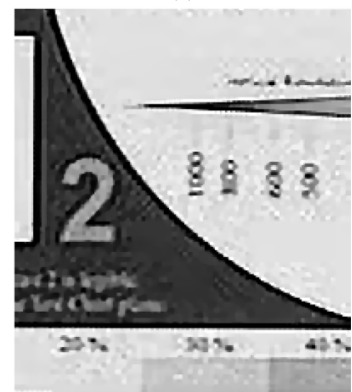

(f)
Fig. 13. Super resolution results (3x resolution increase) by (a) bicubic interpolation, (b) $E F$, (c) $R S R$, (d) $N L S$, (e) $A L G 1$ and (f) $A L G 2$.

including the noise variances, are modeled within a hierarchical Bayesian framework. Using this model, we develop two algorithms with variational Bayesian analysis, both of which estimate all unknowns and algorithm parameters solely from the observed low resolution images without prior knowledge or user intervention. We have shown that the proposed motion estimation method generalizes the classical Lucas-Kanade registration method in a stochastic sense. The proposed methods have the following advantages: First, the proposed framework allows for estimation of distributions of unknowns, which prevent the propagation of estimation errors within the estimation procedure. This is especially useful when the acquisition noise is heavy. Second, through the incorporation of motion estimation and adaptive estimation of the algorithm parameters, the algorithms are very robust to errors in motion estimates. Third, all required parameters of the algorithms are calculated automatically so they do not require user supervision unlike most existing super resolution methods. Experimental results with both synthetic and real images demonstrate that despite the lack of manual parameter tuning, the proposed methods provide super resolution results superior to existing algorithms. Finally, we have shown that the proposed framework can be extended to 


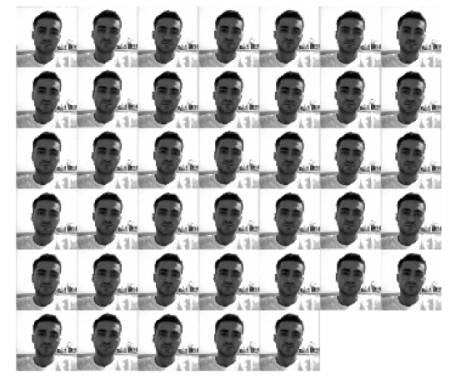

(a)

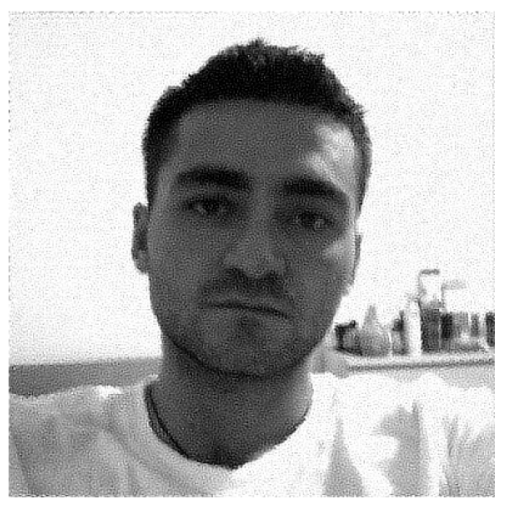

(d)

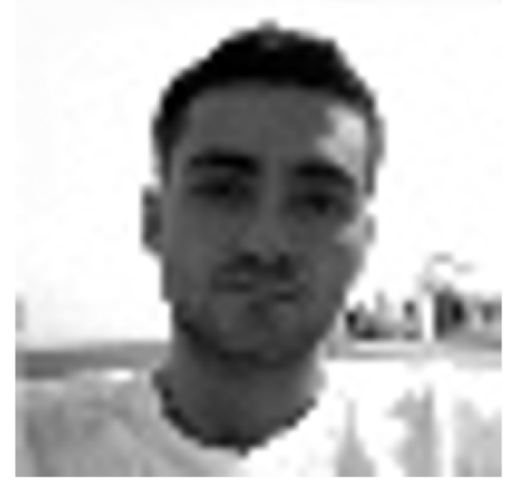

(b)

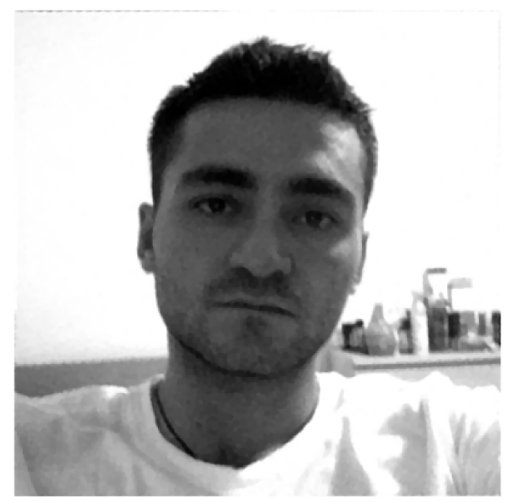

(e)

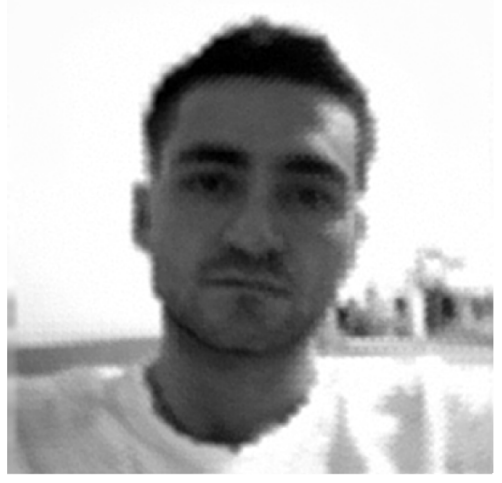

(c)

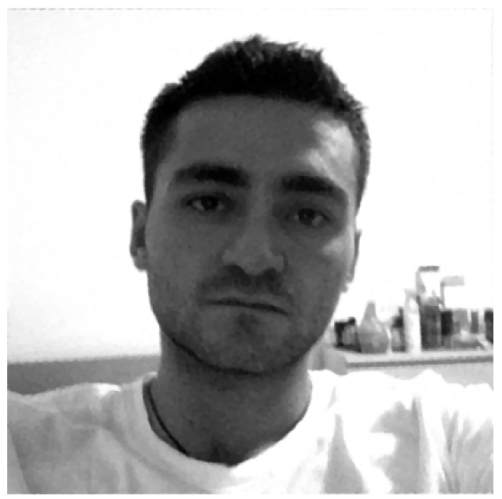

(f)

Fig. 14. Super resolution results (8x resolution increase) on a real dataset. (a) 40 LR images, and the results of (b) bicubic interpolation, (c) $R S R$, (d) NLS, (e) $A L G 1$ and (f) $A L G 2$.

more general super resolution applications with more complex motion models.

\section{REFERENCES}

[1] A. K. Katsaggelos, R. Molina, and J. Mateos, Super Resolution of Images and Video. San Rafael, CA: Morgan and Claypool, 2007.

[2] M. Kang and S. Chaudhuri, Eds., "Super-resolution image reconstruction," IEEE Signal Process. Mag., vol. 20, no. 3, pp. 19-20, 2003.

[3] M. Ng, T. Chan, M. Kang, and P. Milanfar, "Super-resolution imaging: Analysis, algorithms, and applications," EURASIP J. Appl. Signal Process., vol. 2006, 2006, Article ID 90531.

[4] M. Elad and A. Feuer, "Restoration of a single superresolution image from several blurred, noisy, and undersampled measured images," IEEE Trans. Image Process., vol. 6, no. 12, pp. 1646-1658, Dec. 1997.

[5] A. Zomet, A. Rav-Acha, and S. Peleg, "Robust super-resolution," in Proc. IEEE CVPR 2001, 2001, pp. 645-650.

[6] S. Farsiu, M. D. Robinson, M. Elad, and P. Milanfar, "Fast and robust multiframe super resolution," IEEE Trans. Image Process., vol. 13, no. 10, pp. 1327-1344, Oct. 2004.

[7] P. Vandewalle, L. Sbaiz, J. Vandewalle, and M. Vetterli, "Super-resolution from unregistered and totally aliased signals using subspace methods," IEEE Trans. Signal Processing, vol. 55, no. 7, pt. 2, pp. 3687-3703, Jul. 2007.

[8] E. S. Lee and M. G. Kang, "Regularized adaptive high-resolution, image reconstruction considering inaccurate subpixel registration," IEEE Trans. Image Process., vol. 12, no. 7, pp. 826-837, Jul. 2003.

[9] H. He and L. Kondi, "An image super-resolution algorithm for different error levels per frame," IEEE Trans. Image Process., vol. 15, no. 3, pp. 592-603, Mar. 2006.

[10] R. Hardie, K. Barnard, and E. Armstrong, "Joint MAP registration and high-resolution image estimation using a sequence of undersampled images," IEEE Trans. Image Process., vol. 6, no. 12, pp. 1621-1633, 1997.
[11] M. Ng, J. Koo, and N. Bose, "Constrained total least-squares computations for high-resolution image reconstruction with multisensors," Int. J. Imag. Syst. Technol., vol. 12, no. 1, pp. 35-42, 2002.

[12] C. Segall, R. Molina, and A. Katsaggelos, "High-resolution images from low-resolution compressed video," IEEE Signal Process. Mag., vol. 20, pp. 37-48, 2003.

[13] F. Sroubek and J. Flusser, "Multichannel blind deconvolution of spatially misaligned images," IEEE Trans. Image Process., vol. 7, pp. 45-53, Jul. 2005.

[14] N. Woods, N. Galatsanos, and A. Katsaggelos, "Stochastic methods for joint registration, restoration, and interpolation of multiple undersampled images," IEEE Trans. Image Process., vol. 15, pp. 201-213, 2006.

[15] A. Kanemura, S.-I. Maeda, and S. Ishii, "Superresolution with compound Markov random fields via the variational EM algorithm," Neural Networks, vol. 22, no. 7, pp. 1025-1034, 2009.

[16] M. E. Tipping and C. M. Bishop, "Bayesian image super-resolution," in Advances in Neural Information Processing Systems 15 (NIPS). Cambridge: MIT Press, 2003.

[17] L. C. Pickup, D. P. Capel, S. J. Roberts, and A. Zisserman, "Bayesian methods for image super-resolution," Comput. J., 2007.

[18] H. Shen, L. Zhang, B. Huang, and P. Li, "A MAP approach for joint motion estimation, segmentation, and super resolution," IEEE Trans. Image Process., vol. 16, no. 2, pp. 479-490, Feb. 2007.

[19] B. C. Tom and A. K. Katsaggelos, "Reconstruction of a high-resolution image by simultaneous registration, restoration, and interpolation of low-resolution images," in Proc. IEEE ICIP, Washington, DC, 1995, p. 2539.

[20] B. C. Tom, A. K. Katsaggelos, and N. P. Galatsanos, "Reconstruction of a high resolution image from registration and restoration of low resolution images," in Proc. IEEE ICIP, Austin, TX, Jan. 1994, pp. $553-557$.

[21] Y. He, K. H. Yap, L. Chen, and L. P. Chau, "A nonlinear least square technique for simultaneous image registration and super-resolution," IEEE Trans. Image Process., vol. 16, no. 11, pp. 2830-2841, Nov. 2007. 
[22] J. Chung, E. Haber, and J. Nagy, "Numerical methods for coupled super-resolution," Inverse Problems, vol. 22, no. 4, pp. 1261-1272, Aug. 2006.

[23] D. Robinson, S. Farsiu, and P. Milanfar, "Optimal registration of aliased images using variable projection with applications to super-resolution," Comput. J., vol. 52, no. 1, pp. 31-42, 2009.

[24] H. Takeda, P. Milanfar, M. Protter, and M. Elad, "Super-resolution without explicit subpixel motion estimation," IEEE Trans. Image Process., vol. 18, no. 9, pp. 1958-1975, Sep. 2009.

[25] M. Protter, M. Elad, H. Takeda, and P. Milanfar, "Generalizing the nonlocal-means to super-resolution reconstruction," IEEE Trans. Image Process., vol. 18, no. 1, pp. 36-51, Jan. 2009.

[26] S. Baker and T. Kanade, "Hallucinating faces," in Proc. 4th Int. Conf. Automatic Face Gesture Recognit., 2000.

[27] W. T. Freeman, T. R. Jones, and E. C. Pasztor, "Example-based superresolution," IEEE Comput. Graph. Appl., vol. 22, no. 2, pp. 56-65, 2002.

[28] T. F. Chan, M. K. Ng, A. C. Yau, and A. M. Yip, "Superresolution image reconstruction using fast inpainting algorithms," Appl. Comput. Harmonic Anal. (Special Issue on Mathematical Imaging), vol. 23, no. 1, pp. 3-24, 2007.

[29] L. I. Rudin, S. Osher, and E. Fatemi, "Nonlinear total variation based noise removal algorithms," Physica D pp. 259-268, 1992.

[30] S. Babacan, R. Molina, and A. Katsaggelos, "Variational Bayesian blind deconvolution using a total variation prior," IEEE Trans. Image Process., vol. 18, no. 1, pp. 12-26, Jan. 2009.

[31] M. K. Ng, H. Shen, E. Y. Lam, and L. Zhang, "A total variation regularization based super-resolution reconstruction algorithm for digital video," EURASIP J. Adv. Signal Process., no. 74585, 2007.

[32] P. Vandewalle, S. Ssstrunk, and M. Vetterli, "A frequency domain approach to registration of aliased images with application to super-resolution," EURASIP J. Appl. Signal Process. (Special Issue on Super-resolution), vol. 2006, 2006, pp. Article ID 71 459, 14 pages.

[33] B. Lucas and T. Kanade, "An iterative image registration technique with an application to stereo vision," in Proc. Imaging Understanding Workshop, 1981, pp. 121-130.

[34] J. O. Berger, Statistical Decision Theory and Bayesian Analysis. New York: Springer Verlag, 1985, ch. 3 and 4.

[35] C. Bishop, Pattern Recognition and Machine Learning. New York: Springer, 2006.

[36] M. Beal, "Variational algorithms for approximate bayesian inference," Ph.D. dissertation, The Gatsby Computational Neuroscience Unit, University College London, U.K., 2003.

[37] J. Miskin, "Ensemble learning for independent component analysis," Ph.D. dissertation, Astrophysics Group, Univ. Cambridge, U.K., 2000.

[38] S. D. Babacan, R. Molina, and A. Katsaggelos, "Parameter estimation, in TV image restoration using variational distribution approximation," IEEE Trans. Image Process., vol. 17, no. 3, pp. 326-339, Mar. 2008.

[39] S. Baker and I. Matthews, "Lucas-kanade 20 years on: A unifying framework," Int. J. Comput. Vis., vol. 56, no. 3, pp. 221-255, Mar. 2004.

[40] C. M. Bishop, Pattern Recognition and Machine Learning. New York: Springer-Verlag, 2006.

[41] G. H. Golub and C. F. Van Loan, Matrix Computations (Johns Hopkins Studies in Mathematical Sciences). Baltimore, MD: The Johns Hopkins University Press, Oct. 1996.

[42] P. Vandewalle, P. Zbinden, and C. Perez, Superresolution v2.0 2006 [Online]. Available: http://lcavwww.epfl.ch/software/superresolution/ index.html

[43] MDSP Super-Resolution and Demosaicing Datasets [Online]. Available: http://www.soe.ucsc.edu/milanfar/software/sr-datasets.html 2007

[44] S. Farsiu, MDSP Resolution Enhancement Software Univ. Calif.. Santa Cruz, 2004.

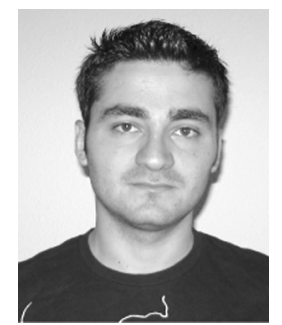

S. Derin Babacan (S'02-M'04) was born in Istanbul, Turkey, in 1981. He received the B.Sc. degree from Bogazici University, Istanbul, in 2004 and the M.Sc. and Ph.D. degrees from Northwestern University, Evanston, IL, in 2006 and 2009, respectively, in the Department of Electrical Engineering and Computer Science.

$\mathrm{He}$ is currently a Beckman Postdoctoral Fellow with the Beckman Institute for Advanced Science and Technology, University of Illinois at Urbana-Champaign. His primary research interests are inverse problems in image processing, computer vision and computational photography.

Dr. Babacan is the recipient of an IEEE International Conference on Image Processing Paper Award (2007).

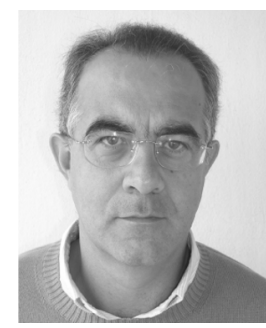

Rafael Molina (M'88) was born in 1957. He received the degree in mathematics (statistics) in 1979 and the Ph.D. degree in optimal design in linear models in 1983 from the University of Granada, Granada, Spain.

He became Professor of computer science and artificial intelligence at the University of Granada, in 2000. His areas of research interest are image restoration (applications to astronomy and medicine), parameter estimation in image restoration, super resolution of images and video, blind deconvolution, and

compressed sensing.

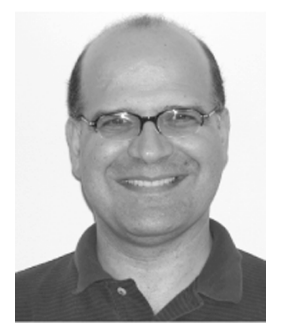

Aggelos K. Katsaggelos (M'85-SM'92-F'98) received the Diploma degree in electrical and mechanical engineering from the Aristotelian University of Thessaloniki, Greece, in 1979 and the M.S. and $\mathrm{Ph} . \mathrm{D}$. degrees both in electrical engineering from the Georgia Institute of Technology, in 1981 and 1985 , respectively.

In 1985 he joined the Department of Electrical and Computer Engineering at Northwestern University, where he is currently professor. He was the holder of the Ameritech Chair of Information Technology (1997-2003). He is also the Director of the Motorola Center for Seamless Communications a member of the Academic Affiliate Staff, Department of Medicine, at Evanston Hospital, and an STA at Argonne National Laboratory. He has published extensively in the areas of signal processing, multimedia transmission, and computer vision. He is the editor of Digital Image Restoration (SpringerVerlag 1991), coauthor of Rate-Distortion Based Video Compression (Kluwer 1997), co-editor of Recovery Techniques for Image and Video Compression and Transmission, (Kluwer 1998), and coauthor of Super-resolution for Images and Video (Claypool, 2007) and Joint Source-Channel Video Transmission (Claypool, 2007).

Dr. Katsaggelos has served the IEEE and other Professional Societies in many capacities (for example, he was editor-in-chief of the IEEE Signal Processing Magazine (1997-2002), a member of the Board of Governors of the IEEE Signal Processing Society (1999-2001), and a member of the Publication Board of the IEEE Proceedings (2003-2007)). He is the co-inventor of 14 international patents, a Fellow of SPIE, and the recipient of the IEEE Third Millennium Medal (2000), the IEEE Signal Processing Society Meritorious Service Award (2001), an IEEE Signal Processing Society Best Paper Award (2001), an IEEE International Conference on Multimedia and Expo Paper Award (2006), an IEEE International Conference on Image Processing Paper Award (2007), and an International Symposium on Image and Signal Processing Paper Award (2009), and the IEEE Signal Processing Society Technical Achievement Award (2010). He was a Distinguished Lecturer of the IEEE Signal Processing Society (2007-2008) . 